\title{
Non-Equilibrium Statistical Mechanics of Anharmonic Chains Coupled to Two Heat Baths at Different Temperatures
}

\author{
J.-P. Eckmann ${ }^{1,2}$, C.-A. Pillet ${ }^{3,4}$ and L. Rey-Bellet ${ }^{2}$ \\ ${ }^{1}$ Dépt. de Physique Théorique, Université de Genève, CH-1211 Genève 4, Switzerland \\ ${ }^{2}$ Section de Mathématiques, Université de Genève, CH-1211 Genève 4, Switzerland \\ ${ }^{3}$ PHYMAT, Université de Toulon, F-83957 La Garde Cedex, France \\ ${ }^{4}$ CPT-CNRS Luminy, F-13288 Marseille Cedex 09
}

\begin{abstract}
We study the statistical mechanics of a finite-dimensional non-linear Hamiltonian system (a chain of anharmonic oscillators) coupled to two heat baths (described by wave equations). Assuming that the initial conditions of the heat baths are distributed according to the Gibbs measures at two different temperatures we study the dynamics of the oscillators. Under suitable assumptions on the potential and on the coupling between the chain and the heat baths, we prove the existence of an invariant measure for any temperature difference, i.e., we prove the existence of steady states. Furthermore, if the temperature difference is sufficiently small, we prove that the invariant measure is unique and mixing. In particular, we develop new techniques for proving the existence of invariant measures for random processes on a non-compact phase space. These techniques are based on an extension of the commutator method of Hörmander used in the study of hypoelliptic differential operators.
\end{abstract}




\section{Introduction}

In this paper, we consider the non-equilibrium statistical mechanics of a finite-dimensional nonlinear Hamiltonian system coupled to two infinite heat baths which are at different temperatures. We show that under certain conditions on the initial data the system goes to a unique nonequilibrium steady state.

To put this new result into perspective, we situate it among other results in equilibrium and non-equilibrium statistical mechanics. First of all, for the case of only one heat bath one expects of course "return to equilibrium." This problem has a long history, and a proof of return to equilibrium under quite general conditions on the non-linear small system and its coupling to the heat bath has been recently obtained in [JP1-4].Viewed from context of our present problem, the main simplifying feature of the one-bath problem is that the final state can be guessed, $a$ priori, to be the familiar Boltzmann distribution.

For the case of two heat baths, there are no results of such generality available, among other things precisely because one cannot guess in general what the steady state is going to be. Since we are dealing with systems on a non-compact phase space and without energy conservation, there is nothing like an SRB Ansatz for our problem [GC]. Worse, even the existence of any stationary state is not obvious at all. The only notable exceptions are problems where the small system and its coupling to the heat baths are linear. Then the problem can be formulated in terms of Gaussian measures, and approach to a steady state has been proved in this case in [RLL], [CL] and [OL] for Markovian heat baths and in [SL] for the general case.

Our approach in the present paper will consist in using the spirit of [FKM] and [FK] to give a microscopic derivation of the equations of motion: under suitable assumptions, we will reduce the study of the dynamics of the coupled system (an infinite dimensional Hamiltonian system) to the study of a random finite dimensional dynamical system. However, we will not achieve the generality of [JP1-4]. Each heat bath is an infinite dimensional linear Hamiltonian system, in our case it will be chosen as the classical field theory associated with the wave equation. The small system is a non-linear Hamiltonian system with an arbitrary (but finite) number of degrees of freedom, in our case it is chosen as a chain of anharmonic oscillators with nearest neighbor couplings. The potential must be of quadratic type near infinite energies. The two heat baths are coupled respectively to the first and the last particle of the chain. The initial conditions of the heat baths will be distributed according to thermal equilibrium at inverse temperatures $\beta_{\mathrm{L}}$, $\beta_{\mathrm{R}}$. Integrating the variables of the heat baths leads to a system of random integro-differential equations: the generalized Langevin equations. They differ from the Newton equations of motion by the addition of two kinds of force, on one hand there is a (random) force exerted by the heat baths on the chain of oscillators and on the other hand there is a dissipative force with memory which describe the genuine retro-action from the heat bath on the small system. We will choose the couplings between the baths and the chain such that the random forces exerted by the baths have an exponentially decaying covariance. With this assumption (see [Tr]), the resulting equations are quasi-Markovian. By this, we mean that one can introduce a finite number of auxiliary variables in such a way that the evolution of the chain, together with these variables, is described by a system of Markovian stochastic differential equations.

With this set-up, we are led to a classical problem in probability theory: the study of invariant measures for diffusion processes. For our problem, the main difficulties are as follows: 
the phase space is not compact and the resulting diffusion process is degenerate and not selfadjoint. ${ }^{1}$ The standard techniques used to prove the existence of invariant measures do not seem to work in our case and, in this paper, we develop new methods to solve this problem, which rely on methods of spectral analysis. Our proof of existence is based on a compactness argument, as often in the proof of existence of invariant measures. More precisely we will prove that the generator of the diffusion process, a second order differential operator, given in our problem, has compact resolvent, in a suitably chosen Hilbert space. This is done by generalizing the commutator method of Hörmander, [Hö], used in the study of hypoelliptic operators. Similar methods have been used to study the spectrum of Schrödinger operators with magnetic fields, see $[\mathrm{HM}],[\mathrm{He}]$, and $[\mathrm{HN}]$.

The restriction to a chain is mostly for convenience. Other geometries can be accommodated with our methods, and the number of heat baths is not restricted to two. Furthermore, the techniques developed in this paper can be applied to other interesting models of non-equilibrium statistical mechanics, for example, an electric field acting on a system of particles [R-B, in preparation].

\section{Description of the Model and Derivation of the Effective Equa- tions}

In this section we define a model of two heat baths coupled to a small system, and derive the stochastic equations which describe the time evolution of the small system. The heat baths are classical field theories associated with the wave equation, the small system is a chain of oscillators and the coupling between them is linear in the field.

We begin the description of the model by defining the "small" system. It is a chain of $d$-dimensional anharmonic oscillators. The phase space of the chain is $\mathbf{R}^{2 d n}$ with $n$ and $d$ arbitrary and its dynamics is described by a $\mathcal{C}^{\infty}$ Hamiltonian function of the form

$$
H_{\mathrm{S}}(p, q)=\sum_{j=1}^{n} \frac{p_{j}^{2}}{2}+\sum_{j=1}^{n} U_{j}\left(q_{j}\right)+\sum_{i=1}^{n-1} U_{i}^{(2)}\left(q_{i}, q_{i+1}\right) \equiv \sum_{j=1}^{n} \frac{p_{j}^{2}}{2}+V(q),
$$

where $q=\left(q_{1}, \ldots, q_{n}\right), p=\left(p_{1}, \ldots, p_{n}\right)$, with $p_{i}, q_{i} \in \mathbf{R}^{d}$.

The potential energy will be assumed "quadratic + bounded" in the following sense. We let $\mathcal{F}$ denote the space of $\mathcal{C}^{\infty}$ functions on $\mathbf{R}^{d n}$ such that for all multi-indices $\alpha$ and all $F \in \mathcal{F}$, the quantity $\partial^{\alpha} F(q)$ is bounded uniformly in $q \in \mathbf{R}^{d n}$. Then our hypotheses are

H1) Behavior at infinity: We assume that $V$ is of the form

$$
V(q)=\frac{1}{2}(q-a, \mathbf{Q}(q-a))+F(q),
$$

where $\mathbf{Q}$ is a positive definite $(d n \times d n)$ matrix, $a$ is a vector, and $\partial_{q_{i}^{(\nu)}} F \in \mathcal{F}$ for $i=1, \ldots, n$ and $\nu=1, \ldots, d$.

${ }^{1}$ The diffusion is non-selfadjoint because the diffusion process is not time-reversal invariant. But the generator $L$ satisfies $L^{*} T=T L$ where $T$ changes the sign of the momenta. 
H2) Coupling: Each of the $(d \times d)$ matrices

$$
\mathcal{M}_{i, i+1}(q) \equiv \nabla_{q_{i}} \nabla_{q_{i+1}} U_{i}^{(2)}\left(q_{i}, q_{i+1}\right), \quad i=1, \ldots, n-1,
$$

is either uniformly positive or negative definite.

Remark. The first hypothesis makes sure the particles do not "fly away." The second hypothesis makes sure that the nearest neighbor interaction can transmit energy. As such, this condition is of the hypoelliptic type.

Example. A typical case (in dimension $d$ ) covered by these hypotheses is given by

$$
U_{j}(q)=q^{2}+5 \sin \left(\sqrt{1+q^{2}}\right), \quad U_{i}^{(2)}\left(q, q^{\prime}\right)=\left(q-q^{\prime}\right)^{2}+\sin \left(\sqrt{1+\left(q-q^{\prime}\right)^{2}}\right) /(2 d) .
$$

As a model of a heat bath we consider the classical field theory associated with the $d$ dimensional wave equation. The field $\varphi$ and its conjugate momentum field $\pi$ are elements of the real Hilbert space $\mathcal{H}=\mathrm{H}_{\mathbf{R}}^{1}\left(\mathbf{R}^{d}\right) \oplus \mathbf{L}_{\mathbf{R}}^{2}\left(\mathbf{R}^{d}\right)$ which is the completion of $\mathcal{C}_{0}^{\infty}\left(\mathbf{R}^{d}\right) \oplus \mathcal{C}_{0}^{\infty}\left(\mathbf{R}^{d}\right)$ with respect to the norm defined by the scalar product:

$$
\left(\left(\begin{array}{c}
\varphi \\
\pi
\end{array}\right),\left(\begin{array}{c}
\varphi \\
\pi
\end{array}\right)\right)_{\mathcal{H}}=\int \mathrm{dx}\left(|\nabla \varphi(x)|^{2}+|\pi(x)|^{2}\right) .
$$

The Hamilton function of the free heat bath is

$$
H_{\mathrm{B}}(\varphi, \pi)=\frac{1}{2} \int \mathrm{dx}\left(|\nabla \varphi(x)|^{2}+|\pi(x)|^{2}\right),
$$

and the corresponding equation of motion is the ordinary wave equation which we write in the form

$$
\left(\begin{array}{c}
\dot{\varphi}(t) \\
\dot{\pi}(t)
\end{array}\right)=\mathcal{L}\left(\begin{array}{c}
\varphi \\
\pi
\end{array}\right)
$$

where

$$
\mathcal{L} \equiv\left(\begin{array}{cc}
0 & 1 \\
\Delta & 0
\end{array}\right)
$$

Let us turn to the coupling between the chain and the heat baths. The baths will be called "L" and "R", the left bath couples to the coordinate $q_{1}$ and the right bath couples to the other end of the chain $\left(q_{n}\right)$. Since we consider two heat baths, the phase space of the coupled system, for finite energy configurations, is $\mathbf{R}^{2 d n} \times \mathcal{H} \times \mathcal{H}$ and its Hamiltonian will be chosen as

$$
\begin{aligned}
H\left(p, q, \varphi_{\mathrm{L}}, \pi_{\mathrm{L}}, \varphi_{\mathrm{R}}, \pi_{\mathrm{R}}\right) & =H_{\mathrm{S}}(p, q)+H_{\mathrm{B}}\left(\varphi_{\mathrm{L}}, \pi_{\mathrm{L}}\right)+H_{\mathrm{B}}\left(\varphi_{\mathrm{R}}, \pi_{\mathrm{R}}\right) \\
& +q_{1} \cdot \int \mathrm{dx} \rho_{\mathrm{L}}(x) \nabla \varphi_{\mathrm{L}}(x)+q_{n} \cdot \int \mathrm{dx} \rho_{\mathrm{R}}(x) \nabla \varphi_{\mathrm{R}}(x) .
\end{aligned}
$$

Here, the $\rho_{j}(x) \in \mathrm{L}^{1}\left(\mathbf{R}^{d}\right)$ are charge densities which we assume for simplicity to be spherically symmetric functions. The choice of the Hamiltonian Eq.(2.3) is motivated by the dipole 
approximation of classical electrodynamics. For notational purposes we use in the sequel the shorthand

$$
\phi_{i} \equiv\left(\begin{array}{c}
\varphi_{i} \\
\pi_{i}
\end{array}\right) .
$$

We set $\alpha_{i}=\left(\alpha_{i}^{(1)}, \ldots, \alpha_{i}^{(d)}\right), i \in\{\mathrm{L}, \mathrm{R}\}$, with

$$
\widehat{\alpha}_{i}^{(\nu)}(k) \equiv\left(\begin{array}{c}
-i k^{(\nu)} \widehat{\rho}_{i}(k) / k^{2} \\
0
\end{array}\right) .
$$

Here and in the sequel the "hat" means the Fourier transform

$$
\widehat{f}(k) \equiv \frac{1}{(2 \pi)^{d / 2}} \int \mathrm{dx} f(x) e^{-i k \cdot x} .
$$

With this notation the Hamiltonian becomes

$$
H\left(p, q, \phi_{\mathrm{L}}, \phi_{\mathrm{R}}\right)=H_{\mathrm{S}}(p, q)+H_{\mathrm{B}}\left(\phi_{\mathrm{L}}\right)+H_{\mathrm{B}}\left(\phi_{\mathrm{R}}\right)+q_{1} \cdot\left(\phi_{\mathrm{L}}, \alpha_{\mathrm{L}}\right)_{\mathcal{H}}+q_{n} \cdot\left(\phi_{\mathrm{R}}, \alpha_{\mathrm{R}}\right)_{\mathcal{H}},
$$

where $H_{\mathrm{B}}(\phi)=\frac{1}{2}\|\phi\|_{\mathcal{H}}^{2}$. We next study the equations of motions. They take the form

$$
\begin{aligned}
\dot{q}_{j}(t) & =p_{j}(t), \quad j=1, \ldots, n, \\
\dot{p}_{1}(t) & =-\nabla_{q_{1}} V(q(t))-\left(\phi_{\mathrm{L}}(t), \alpha_{\mathrm{L}}\right)_{\mathcal{H}}, \\
\dot{p}_{j}(t) & =-\nabla_{q_{j}} V(q(t)), \quad j=2, \ldots, n-1, \\
\dot{p}_{n}(t) & =-\nabla_{q_{n}} V(q(t))-\left(\phi_{\mathrm{R}}(t), \alpha_{\mathrm{R}}\right)_{\mathcal{H}}, \\
\dot{\phi}_{\mathrm{L}}(t) & =\mathcal{L}\left(\phi_{\mathrm{L}}(t)+\alpha_{\mathrm{L}} \cdot q_{1}(t)\right), \\
\dot{\phi}_{\mathrm{R}}(t) & =\mathcal{L}\left(\phi_{\mathrm{R}}(t)+\alpha_{\mathrm{R}} \cdot q_{n}(t)\right) .
\end{aligned}
$$

The last two equations of (2.4) are easily integrated and lead to

$$
\begin{aligned}
& \phi_{\mathrm{L}}(t)=e^{\mathcal{L} t} \phi_{\mathrm{L}}(0)+\int_{0}^{t} \mathrm{~d} s \mathcal{L} e^{\mathcal{L}(t-s)} \alpha_{\mathrm{L}} \cdot q_{1}(s), \\
& \phi_{\mathrm{R}}(t)=e^{\mathcal{L} t} \phi_{\mathrm{R}}(0)+\int_{0}^{t} \mathrm{~d} s \mathcal{L} e^{\mathcal{L}(t-s)} \alpha_{\mathrm{R}} \cdot q_{n}(s),
\end{aligned}
$$

where the $\phi_{i}(0), i \in\{\mathrm{L}, \mathbf{R}\}$, are the initial conditions of the heat baths. Inserting into the first $2 n$ equations of (2.4) gives the following system of integro-differential equations

$$
\begin{aligned}
& \dot{q}_{j}(t)=p_{j}(t), \quad j=1, \ldots, n, \\
& \dot{p}_{1}(t)=-\nabla_{q_{1}} V(q(t))-\left(\phi_{\mathrm{L}}(0), e^{-\mathcal{L} t} \alpha_{\mathrm{L}}\right)_{\mathcal{H}}-\int_{0}^{t} \mathrm{~d} s D_{\mathrm{L}}(t-s) q_{1}(s), \\
& \dot{p}_{j}(t)=-\nabla_{q_{j}} V(q(t)), \quad j=2, \ldots, n-1, \\
& \dot{p}_{n}(t)=-\nabla_{q_{n}} V(q(t))-\left(\phi_{\mathrm{R}}(0), e^{-\mathcal{L} t} \alpha_{\mathrm{R}}\right)_{\mathcal{H}}-\int_{0}^{t} \mathrm{~d} s D_{\mathrm{R}}(t-s) q_{n}(s),
\end{aligned}
$$


where the $d \times d$ dissipation matrices $D_{i}^{(\mu, \nu)}(t-s), i \in\{\mathrm{L}, \mathrm{R}\}$, are given by

$$
\begin{aligned}
D_{i}^{(\mu, \nu)}(t-s) & =\left(\alpha_{i}^{(\mu)}, \mathcal{L} e^{\mathcal{L}(t-s)} \alpha_{i}^{(\nu)}\right)_{\mathcal{H}} \\
& =-\frac{1}{d} \delta_{\mu, \nu} \int \mathrm{dk}\left|\widehat{\rho}_{i}(k)\right|^{2}|k| \sin (|k|(t-s)) .
\end{aligned}
$$

The last expression is obtained by observing that

$$
e^{\mathcal{L} t}=\left(\begin{array}{cc}
\cos (|k| t) & |k|^{-1} \sin (|k| t) \\
-|k| \sin (|k| t) & \cos (|k| t)
\end{array}\right),
$$

written in Fourier space.

So far we only discussed the finite energy configurations of the heat baths. We will assume that the two reservoirs are in thermal equilibrium at inverse temperatures $\beta_{\mathrm{L}}$ and $\beta_{\mathrm{R}}$. This means that the initial conditions $\Phi(0) \equiv\left\{\phi_{\mathrm{L}}(0), \phi_{\mathrm{R}}(0)\right\}$ are distributed according to the Gaussian measure with mean zero and covariance $\left\langle\phi_{i}(f) \phi_{j}(g)\right\rangle=\delta_{i j}\left(1 / \beta_{i}\right)(f, g)_{\mathcal{H}}$. (Recall that the Hamiltonian of the heat baths is given by $\sum_{i \in\{\mathrm{L}, \mathrm{R}\}}\left(\phi_{i}, \phi_{i}\right)_{\mathcal{H}} \cdot$ ) If we assume that the coupling functions $\alpha_{i}^{(\nu)}$ are in $\mathcal{H}, i \in\{\mathrm{L}, \mathrm{R}\}$, and $\nu \in\{1, \cdots, d\}$ then the $\xi_{i}(t) \equiv \phi_{i}(0)\left(e^{-\mathcal{L} t} \alpha_{i}\right)$ become $d$-dimensional Gaussian random processes with mean zero and covariance

$$
\left\langle\xi_{i}(t) \xi_{j}(s)\right\rangle=\delta_{i, j} \frac{1}{\beta_{i}} C_{i}(t-s), \quad i, j \in\{\mathrm{L}, \mathrm{R}\},
$$

and the $d \times d$ covariance matrices $C_{i}(t-s)$ are given by

$$
\begin{aligned}
C_{i}^{(\mu, \nu)}(t-s) & =\left(\alpha_{i}^{(\mu)}, e^{\mathcal{L}(t-s)} \alpha_{i}^{(\nu)}\right)_{\mathcal{H}} \\
& =\frac{1}{d} \delta_{\mu, \nu} \int \mathrm{dk}\left|\widehat{\rho}_{i}(k)\right|^{2} \cos (|k|(t-s)) .
\end{aligned}
$$

The relation

$$
\dot{C}_{i}(t)=D_{i}(t)
$$

which is checked easily by inspection, is known as the fluctuation dissipation theorem. It is characteristic of the Hamiltonian nature of the system. After these assumptions and transformations, the equations of motion (2.5) become a system of random integro-differential equations on $\mathbf{R}^{2 d n}$ which we will analyze in the sequel.

Finally, we impose a condition on the random force exerted by the heat baths on the chain. We assume that

H3) The covariances of the random processes $\xi_{i}(t)$ with $i \in\{\mathrm{L}, \mathrm{R}\}$ satisfy $C_{i}^{(\mu, \nu)}(t-s)=$ $\delta_{\mu, \nu} \sum_{m=1}^{M} \lambda_{i, m}^{2} e^{-\gamma_{i, m}|t-s|}$, with $\gamma_{i, m}>0$ and $\lambda_{i, m}>0$.

This can be achieved by a suitable choice of the coupling functions $\rho_{i}(x)$, for example

$$
\widehat{\rho}_{i}(k)=\text { const. } \prod_{m=1}^{M} \frac{1}{\left(k^{2}+\gamma_{i, m}^{2}\right)^{1 / 2}},
$$


where all the $\gamma_{i, m}$ are distinct. To keep the notation from still further accumulating, we choose $M$ the same on the left and the right. We will call the random process given by Eq.(2.5) quasi-Markovian if Condition H3 is satisfied. Indeed, using Condition H3 together with the fluctuation-dissipation relation (2.7) and enlarging the phase space one may eliminate the memory terms (both deterministic and random) of the equations of motion (2.5) and rewrite them as a system of Markovian stochastic differential equations.

By Condition $\mathbf{H 3}$ we can rewrite the stochastic processes $\xi_{i}(t)$ as Itô stochastic integrals

$$
\xi_{i}(t)=\sum_{m=1}^{M} \lambda_{i, m} \sqrt{\frac{2 \gamma_{i, m}}{\beta_{i}}} \int_{-\infty}^{t} e^{-\gamma_{i, m}(t-s)} \mathrm{d} w_{i, m}(s),
$$

where the $w_{i, m}(s)$ are $d$-dimensional Wiener processes with covariance

$$
\mathbf{E}\left[\left(w_{i, m}^{(\mu)}(t)-w_{i, m}^{(\mu)}(s)\right)\left(w_{j, m^{\prime}}^{(\nu)}\left(t^{\prime}\right)-w_{j, m^{\prime}}^{(\nu)}\left(s^{\prime}\right)\right)\right]=\delta_{i, j} \delta_{\mu, \nu} \delta_{m, m^{\prime}}\left|[s, t] \cap\left[s^{\prime}, t^{\prime}\right]\right|,
$$

where $s<t$ and $s^{\prime}<t^{\prime}, \mathbf{E}$ is the expectation on the probability space of the Wiener process and $|\cdot|$ denotes the Lebesgue measure. We introduce new "effective" variables $r_{\mathrm{L}, m}, r_{\mathrm{R}, m} \in \mathbf{R}^{d}$, with $m=1, \ldots, M$, which describe both the retro-action of the heat bath onto the system and the random force exerted by the heat baths:

$$
\begin{aligned}
& r_{\mathrm{L}, m}(t)=\lambda_{\mathrm{L}, m}^{2} \gamma_{\mathrm{L}, m} \int_{0}^{t} \mathrm{~d} s e^{-\gamma_{\mathrm{L}, m}(t-s)} q_{1}(s)-\lambda_{\mathrm{L}, m} \sqrt{\frac{2 \gamma_{\mathrm{L}, m}}{\beta_{\mathrm{L}}}} \int_{-\infty}^{t} e^{-\gamma_{\mathrm{L}, m}(t-s)} \mathrm{d} w_{\mathrm{L}, m}(s), \\
& r_{\mathrm{R}, m}(t)=\lambda_{\mathrm{R}, m}^{2} \gamma_{\mathrm{R}, m} \int_{0}^{t} \mathrm{~d} s e^{-\gamma_{\mathrm{R}, m}(t-s)} q_{n}(s)-\lambda_{\mathrm{R}, m} \sqrt{\frac{2 \gamma_{\mathrm{R}, m}}{\beta_{\mathrm{R}}}} \int_{-\infty}^{t} e^{-\gamma_{\mathrm{R}, m}(t-s)} \mathrm{d} w_{\mathrm{R}, m}(s) .
\end{aligned}
$$

We get the following system of Markovian stochastic differential equations

$$
\begin{aligned}
\mathrm{d} q_{j}(t) & =p_{j}(t) \mathrm{d} t, \quad j=1, \ldots, n, \\
\mathrm{~d} p_{1}(t) & =-\nabla_{q_{1}} V(q(t)) \mathrm{d} t+\sum_{m=1}^{M} r_{\mathrm{L}, m}(t) \mathrm{d} t, \\
\mathrm{~d} p_{j}(t) & =-\nabla_{q_{j}} V(q(t)) \mathrm{d} t, \quad j=2, \ldots, n-1, \\
\mathrm{~d} p_{n}(t) & =-\nabla_{q_{n}} V(q(t)) \mathrm{d} t+\sum_{m=1}^{M} r_{\mathrm{R}, m}(t) \mathrm{d} t, \\
\mathrm{~d} r_{\mathrm{L}, m}(t) & =-\gamma_{\mathrm{L}, m} r_{\mathrm{L}, m}(t) \mathrm{d} t+\lambda_{\mathrm{L}, m}^{2} \gamma_{\mathrm{L}, m} q_{1}(t) \mathrm{d} t-\lambda_{\mathrm{L}, m} \sqrt{\frac{2 \gamma_{\mathrm{L}, m}}{\beta_{\mathrm{L}}}} \mathrm{d} w_{\mathrm{L}, m}(t), \\
\mathrm{d} r_{\mathrm{R}, m}(t) & =-\gamma_{\mathrm{R}, m} r_{\mathrm{R}, m}(t) \mathrm{d} t+\lambda_{\mathrm{R}, m}^{2} \gamma_{\mathrm{R}, m} q_{n}(t) \mathrm{d} t-\lambda_{\mathrm{R}, m} \sqrt{\frac{2 \gamma_{\mathrm{R}, m}}{\beta_{\mathrm{R}}}} \mathrm{d} w_{\mathrm{R}, m}(t),
\end{aligned}
$$


which defines a Markov diffusion process on $\mathbf{R}^{2 d(n+M)}$. This system of equations is our main object of study. Our main results are the following:

Theorem 2.1. If Conditions H1-H2 hold, there is a constant $\lambda^{*}>0$, such that for $\left|\lambda_{\mathrm{L}, m}\right|$, $\left|\lambda_{\mathrm{R}, m}\right| \in\left(0, \lambda^{*}\right)$ with $m=1, \ldots, M$, the solution of Eq.(2.9) is a Markov process which has an absolutely continuous invariant measure $\mu$ with a $\mathcal{C}^{\infty}$ density.

Remark. In Proposition 3.6 we will show even a little more. Let $h_{0}(\beta)$ be the Gibbs distribution for our system when the heat baths are both at temperature $1 / \beta$. If $h$ denotes the density of the invariant measure found in Theorem 2.1, we find that $h / h_{0}(\beta)$ is in the Schwartz space $\mathcal{S}$ for all $\beta<\min \left(\beta_{\mathrm{L}}, \beta_{\mathrm{R}}\right)$. This mathematical statement reflects the intuitively obvious fact that the chain can not get hotter than either of the baths.

Concerning the uniqueness and the ergodic properties of the invariant measure, our results are restricted to small temperature differences. We have the following result.

Theorem 2.2. If Conditions H1-H2 hold, there are constants $\lambda^{*}>0$ and $\varepsilon>0$ such that for $\left|\lambda_{\mathrm{L}, m}\right|,\left|\lambda_{\mathrm{R}, m}\right| \in\left(0, \lambda^{*}\right)$ with $m=1, \ldots, M$, and $\left|\beta_{\mathrm{L}}-\beta_{\mathrm{R}}\right| /\left(\beta_{\mathrm{L}}+\beta_{\mathrm{R}}\right)<\varepsilon$, the Markov process (2.9) has a unique invariant measure and this measure is mixing.

Remark. The restriction on the couplings between the small system and the baths $\lambda_{\mathrm{L}, m}, \lambda_{\mathrm{R}, m}$ is non-perturbative: it is a condition of stability of the coupled system small system plus heat baths. Indeed, the baths have the effect of renormalizing the deterministic potential seen by the small system. The constant $\lambda^{*}$ depends only on the potential $V(q)$ : if the coupling constants $\lambda_{\mathrm{L}, m}, \lambda_{\mathrm{R}, m}$ are too large, the effective potential ceases to be stable and, at least at equilibrium (i.e., for $\beta_{\mathrm{L}}=\beta_{\mathrm{R}}$ ), there is no invariant probability measure for the Markov process (2.9), but only a $\sigma$-finite invariant measure (see Eq.(3.7) and Eq.(3.9)). This restriction is related to Condition $\mathbf{H 1}$ on the potential: for potentials which grow at infinity faster than quadratically, this restriction would not be present (see [JP1]). On the other hand, the restriction on the temperature difference $i$ s of perturbative origin.

A more physical formulation of the results of Theorem 2.2 is obtained by going back to the Eq.(2.5), which expresses all the quantities in terms of the phase space of the small system and the initial conditions $\Phi(0)$ of the heat baths. Let us introduce some notation: For given initial conditions $\Phi(0)$, we let $\Theta_{t, \Phi(0)}(p, q)$ denote the solution of Eq.(2.5). Finally, define

$$
\nu(\mathrm{dp}, \mathrm{dq})=\int_{r \in \mathbf{R}^{2 d M}} \mu(\mathrm{dp}, \mathrm{dq}, \mathrm{dr}),
$$

where $\mu$ is the invariant measure of Theorem 2.1.

Corollary 2.3. Under the hypotheses of Theorem 2.2, the system Eq.(2.5) reaches a stationary state and is mixing in the following sense: For any observables $F, G \in \mathrm{L}^{2}\left(\mathbf{R}^{2 d n}, \nu(\mathrm{dp}, \mathrm{dq})\right)$ and for any probability measure $\nu_{0}(\mathrm{dp}, \mathrm{dq})$ which is absolutely continuous with respect to $\nu(\mathrm{dp}, \mathrm{dq})$ 
we have

$$
\begin{aligned}
\lim _{t \rightarrow \infty} \int \nu_{0}(\mathrm{dp}, \mathrm{dq})\left\langle\left(F \circ \Theta_{t, \Phi_{0}}\right)(p, q)\right\rangle & =\int \nu(\mathrm{dp}, \mathrm{dq}) F(p, q), \\
\lim _{t \rightarrow \infty} \int \nu(\mathrm{dp}, \mathrm{dq})\left\langle\left(F \circ \Theta_{t, \Phi_{0}}\right)(p, q) G(p, q)\right\rangle & =\int \nu(\mathrm{dp}, \mathrm{dq}) F(p, q) \int \nu(\mathrm{dp}, \mathrm{dq}) G(p, q) .
\end{aligned}
$$

Here, $\langle\cdot\rangle$ denotes the integration over the Gaussian measures of the two heat baths, introduced earlier.

We explain next the strategy to prove these results. Our proof is based on a detailed study of Eq.(2.9). Let $x=(p, q, r)$. For a Markov process $x(t)$ with phase space $X$ and an invariant measure $\mu(\mathrm{dx})$, its ergodic properties may be deduced from the study of the associated semigroup $T^{t}$ on the Hilbert space $\mathrm{L}^{2}(X, \mu(\mathrm{dx}))$. To prove the existence of the invariant measure in Theorem 2.1 we proceed as follows: We consider first the semi-group $T^{t}$ on the auxiliary Hilbert space $\mathcal{H}_{0} \equiv \mathrm{L}^{2}\left(X, \mu_{0}(\mathrm{dx})\right)$, where the reference measure $\mu_{0}(\mathrm{dx})$ is a generalized Gibbs state for a suitably chosen reference temperature. Our main technical result consists in proving that the generator $L$ of the semi-group $T^{t}$ on $\mathcal{H}_{0}$ and its adjoint $L^{*}$ have compact resolvent. This is proved by generalizing the commutator method developed by Hörmander to study hypoelliptic operators. From this follows the existence of a solution to the eigenvalue equation $\left(T^{t}\right)^{*} g=g$ in $\mathcal{H}_{0}$ and this implies immediately the existence of an invariant measure. To prove Theorem 2.2 we use a perturbation argument, indeed at equilibrium (i.e., for $\beta_{\mathrm{L}}=\beta_{\mathrm{R}}$ ) the invariant measure is unique and 0 is a simple eigenvalue of the generator $L$ in $\mathcal{H}_{0}$. Using the compactness properties of $L$, we show that 0 is a simple eigenvalue of the generator $L$ in $\mathcal{H}_{0}$ for $\left|\beta_{\mathrm{L}}-\beta_{\mathrm{R}}\right| /\left(\beta_{\mathrm{L}}+\beta_{\mathrm{R}}\right)$ small enough. And this can be used to prove the uniqueness claim of Theorem 2.2, while the mixing properties will be shown by extending the method of [Tr].

This paper is organized as follows: In Section 3 we prove Theorem 2.1 and Theorem 2.2 except for our main estimates Proposition 3.4 and Proposition 3.5 which are proven in Section 4. In Appendices A, B and C, we prove some auxiliary results.

\section{Invariant Measure: Existence and Ergodic Properties}

In this section, our main aim is to prove Theorem 2.1 and Theorem 2.2. We first prove some basic consequences of our Assumptions $\mathbf{H 1}$ and H2. In particular, we define the semigroup $T^{t}$ describing the solutions of Eq.(2.9) on the auxiliary Hilbert space $\mathcal{H}_{0}$ described in the introduction. Furthermore we recall some basic facts on hypoelliptic differential operators. Once these preliminaries are in place, we can attack the proof of Theorem 2.1 and Theorem 2.2 proper. 


\subsection{Existence and fundamental properties of the dynamics}

Let $X=\mathbf{R}^{2 d(n+M)}$ and write the stochastic differential equation (2.9) in the abbreviated form

$$
d x(t)=b(x(t))+\sigma d w(t),
$$

where

(i) $b$ is a $\mathcal{C}^{\infty}$ vector field which satisfies, by Condition $\mathbf{H} 1$,

$$
\sup _{x \in X}\left|\partial^{\alpha} b(x)\right|<\infty,
$$

for any multi-index $\alpha$ such that $|\alpha| \geq 1$. In particular

$$
B \equiv\|\operatorname{div} b\|_{\infty}<\infty .
$$

(ii) $\sigma: \mathbf{R}^{2 d M} \rightarrow X$ is a linear map. We also define

$$
D \equiv \frac{1}{2} \sigma \sigma^{T} \geq 0
$$

(iii) $w \in \mathcal{W} \equiv \mathcal{C}\left(\mathbf{R} ; \mathbf{R}^{2 d M}\right)$ is a standard $2 d M$-dimensional Wiener process.

The Eq.(3.1) is rewritten more precisely as the integral equation

$$
\xi(t, w ; x)=x+\int_{0}^{t} d s b(\xi(s, w ; x))+\sigma(w(t)-w(0)) .
$$

It follows from an elementary contraction argument (see e.g. [Ne], Thm 8.1) that (3.4) has a unique solution

$$
\mathbf{R} \ni t \mapsto x(t)=\xi(t, w ; x) \in \mathcal{C}(\mathbf{R} ; X),
$$

for arbitrary initial condition $x \in X$ and $w \in \mathcal{W}$.

The difference $w(t)-w(0)$ has the statistics of a standard Brownian motion and we denote by $\mathbf{E}[\cdot]$ the corresponding expectation. By well-known results on stochastic differential equations, this induces on $\xi(t, w ; x)$ the statistics of a Markovian diffusion process with generator

$$
\nabla \cdot D \nabla+b(x) \cdot \nabla .
$$

More precisely (see $[\mathrm{Ne}]$ Theorem 8.1): Let $\mathcal{C}_{\infty}(X)$ denote the continuous functions which vanish at infinity with the sup-norm and let $\mathcal{F}^{t}$ be the $\sigma$-field generated by $x$ and $\{w(s)-$ $w(0) ; 0<s \leq t\}$, then for $0 \leq s \leq t$ and $f \in \mathcal{C}_{\infty}(X)$ we have

$$
\mathbf{E}\left[f(x(t)) \mid \mathcal{F}^{s}\right]=T^{t-s} f(x(s)) \quad \text { a.s. },
$$

where $T^{t}$ is a strongly continuous contraction semi-group of positivity preserving operators on $\mathcal{C}_{\infty}(X)$ whose generator reduces to $(3.5)$ on $\mathcal{C}_{0}^{\infty}(X)$. 
In the sequel we denote by $L$ the differential operator (3.5) with domain $D(L)=\mathcal{C}_{0}^{\infty}(X)$.

To prove the existence of an invariant measure we will study the semi-group $T^{t}$ or rather an extension of it on the auxiliary weighted Hilbert space $\mathcal{H}_{0}$ described in the introduction. To define $\mathcal{H}_{0}$ precisely, we consider the "effective Hamiltonian"

$$
G(p, q, r)=H_{\mathrm{S}}(p, q)+\sum_{m=1}^{M}\left(\frac{1}{\lambda_{\mathrm{L}, m}^{2}} \frac{r_{\mathrm{L}, m}^{2}}{2}+\frac{1}{\lambda_{\mathrm{R}, m}^{2}} \frac{r_{\mathrm{R}, m}^{2}}{2}-q_{1} \cdot r_{\mathrm{L}, m}-q_{n} \cdot r_{\mathrm{R}, m}\right) .
$$

We note that, due to Condition H1, $G(x) \rightarrow+\infty$ as $|x| \rightarrow \infty$ as long as $\left|\lambda_{\mathrm{L}, m}\right|,\left|\lambda_{\mathrm{R}, m}\right|<\lambda^{*}$ for some $\lambda^{*}$ depending only on the potential $V(q)$.

We choose further a "reference temperature" $\beta_{0}$, which is arbitrary subject to the condition

$$
\beta_{0}<2 \min \left(\beta_{\mathrm{L}}, \beta_{\mathrm{R}}\right) \text {. }
$$

For example we could take $\beta_{0}$ as the inverse of the mean temperature of the heat baths: $\beta_{0}^{-1}=\left(\beta_{\mathrm{L}}^{-1}+\beta_{\mathrm{R}}^{-1}\right) / 2$. For the time being, it will be convenient not to fix $\beta_{0}$. Then, we let

$$
\mathcal{H}_{0}=\mathrm{L}^{2}\left(X, Z_{0}^{-1} e^{-\beta_{0} G} \mathrm{dx}\right),
$$

and we denote $(\cdot, \cdot)_{\mathcal{H}_{0}}$ and $\|\cdot\|_{\mathcal{H}_{0}}$ the corresponding scalar product and norm.

Remark. With a proper choice of $Z_{0}$, it is easy to check that the quantity $Z_{0}^{-1} e^{-\beta_{0} G(q, p, r)} \mathrm{dx}$ is the invariant measure for the Markov process Eq.(2.9) when $\beta_{\mathrm{L}}=\beta_{\mathrm{R}}=\beta_{0}$ and $\left|\lambda_{\mathrm{L}, m}\right|,\left|\lambda_{\mathrm{R}, m}\right|<$ $\lambda^{*}$.

Lemma 3.1. If the potential $V$ satisfies Condition $\mathbf{H 1}$ and if $\beta_{0}<2 \min \left(\beta_{\mathrm{L}}, \beta_{\mathrm{R}}\right)$ there is a $\lambda^{*}>0$ such that if the couplings satisfy $\left|\lambda_{\mathrm{L}, m}\right|,\left|\lambda_{\mathrm{R}, m}\right| \in\left(0, \lambda^{*}\right)$, then the semi-group $T^{t}$ given by Eq.(3.6) extends to a strongly continuous quasi bounded semi-group $T_{\mathcal{H}_{0}}^{t}$ on $\mathcal{H}_{0}$ :

$$
\left\|T_{\mathcal{H}_{0}}^{t}\right\|_{\mathcal{H}_{0}} \leq e^{\alpha t}
$$

where $\alpha$ is given by

$\alpha=d \sum_{m=1}^{M}\left(\gamma_{\mathrm{L}, m}\left(\frac{1}{2}-\frac{\sqrt{\left(\beta_{\mathrm{L}}-\beta_{0} / 2\right) \beta_{0} / 2}}{\beta_{\mathrm{L}}}\right)+\gamma_{\mathrm{R}, m}\left(\frac{1}{2}-\frac{\sqrt{\left(\beta_{\mathrm{R}}-\beta_{0} / 2\right) \beta_{0} / 2}}{\beta_{\mathrm{R}}}\right)\right) \geq 0$.

The generator $L_{\mathcal{H}_{0}}$ of $T_{\mathcal{H}_{0}}^{t}$ is the closure of the differential operator $L$ with domain $\mathcal{C}_{0}^{\infty}$ given by

$$
\begin{aligned}
L & =\sum_{m=1}^{M} \frac{\lambda_{\mathrm{L}, m}^{2} \gamma_{\mathrm{L}, m}}{\beta_{\mathrm{L}}}\left(\nabla_{r_{\mathrm{L}, m}}-\beta_{\mathrm{L}} W_{\mathrm{L}, m}\right) \cdot \nabla_{r_{\mathrm{L}, m}} \\
& +\sum_{m=1}^{M} \frac{\lambda_{\mathrm{R}, m}^{2} \gamma_{\mathrm{R}, m}}{\beta_{\mathrm{R}}}\left(\nabla_{r_{\mathrm{R}, m}}-\beta_{\mathrm{R}} W_{\mathrm{R}, m}\right) \cdot \nabla_{r_{\mathrm{R}, m}} \\
& +\sum_{m=1}^{M} r_{\mathrm{L}, m} \cdot \nabla_{p_{1}}+L_{\mathrm{S}}+\sum_{m=1}^{M} r_{\mathrm{R}, m} \cdot \nabla_{p_{n}},
\end{aligned}
$$


with the abbreviations

$$
W_{\mathrm{L}, m}=\lambda_{\mathrm{L}, m}^{-2} r_{\mathrm{L}, m}-q_{1}, \quad W_{\mathrm{R}, m}=\lambda_{\mathrm{R}, m}^{-2} r_{\mathrm{R}, m}-q_{n},
$$

and where $L_{\mathrm{S}}$ is the Liouville operator associated with the Hamiltonian $H_{\mathrm{S}}(q, p)$ :

$$
L_{\mathrm{S}}=\sum_{j=1}^{n} p_{j} \cdot \nabla_{q_{j}}-\left(\nabla_{q_{j}} V\right) \cdot \nabla_{p_{j}} .
$$

Moreover, $T_{\mathcal{H}_{0}}^{t}$ is positivity preserving:

$$
T_{\mathcal{H}_{0}}^{t} f \geq 0 \quad \text { if } \quad f \geq 0
$$

and

$$
T_{\mathcal{H}_{0}}^{t} 1=1 \text {. }
$$

Remark. We have $\alpha=0$ if only if $\beta_{\mathrm{L}}=\beta_{\mathrm{R}}=\beta_{0}$.

Proof. The proof uses standard tools of stochastic analysis and is given in Appendix A.

Having shown a priori bounds using Condition H1, we will state one basic consequence of Condition H2. We recall that a differential operator $P$ is called hypoelliptic if

$$
\operatorname{sing} \operatorname{supp} u=\operatorname{sing} \operatorname{supp} P u \quad \text { for all } u \in \mathcal{D}^{\prime}(X) \text {. }
$$

Here $\mathcal{D}^{\prime}(X)$ is the usual space of distributions on the infinitely differentiable functions with compact support and for $u \in \mathcal{D}^{\prime}(X)$, sing supp $u$ is the set of points $x \in X$ such that there is no open neighborhood of $x$ to which the restriction of $u$ is a $\mathcal{C}^{\infty}$ function.

Let $P$ be of the form

$$
P=\sum_{j=1}^{J} Y_{j}^{2}+Y_{0},
$$

where $Y_{j}, j \in\{0, \ldots, J\}$ are real $\mathcal{C}^{\infty}$ vector fields. Then by Hörmander's Theorem, [Hö], Thm 22.2.1, if the Lie algebra generated by $Y_{j}, j \in\{0, \ldots, J\}$ has rank $\operatorname{dim} X$ at every point, then $P$ is hypoelliptic.

Differential operators arising from diffusion problems are of the form (3.16). Let $L$ be the differential operators given in Eq.(3.11), let $L^{T}$ denote its formal adjoint, then one may easily check that Condition $\mathbf{H 2}$ implies that any of the following operators

$$
L, \quad L^{T}, \quad \partial_{t}+L, \quad \partial_{t}+L^{T},
$$

satisfies the condition of Hörmander's Theorem and thus is hypoelliptic. As an immediate consequence we have: 
Corollary 3.2. If Condition $\mathrm{H} 2$ is satisfied then the eigenvectors of $L$ and $L^{T}$ are $\mathcal{C}^{\infty}$ functions.

Next, let $P(t, x, E), t \geq 0, x \in X, E \in \mathcal{B}$ denote the transition probabilities of the Markov process $\xi(t, w ; x)$ solving the stochastic differential equation (2.9) with initial condition $x$, i.e.,

$$
P(t, x, E)=\mathbf{P}(\xi(t, w: x) \in E),
$$

where $\mathbf{P}$ denotes the probability associated with the Wiener process. Then by the forward and backward Kolmogorov equations we obtain

Corollary 3.3. If Conditions $\mathbf{H 1}$ and $\mathbf{H 2}$ are satisfied then the transition probabilities of the Markov Process $\xi(t, w ; x)$ have a smooth density

$$
P(t, x, y) \in \mathcal{C}^{\infty}((0, \infty) \times X \times X)
$$

\subsection{Proof of Theorem 2.1 and Theorem 2.2}

After these preliminaries we now turn to the study of spectral properties of the generator $L_{\mathcal{H}_{0}}$ of the semi-group $T_{\mathcal{H}_{0}}^{t}$.

The proof of the existence of the invariant measure will be a consequence of the following key property which we prove in Section 4.

Proposition 3.4. If the potential $V$ satisfies Conditions $\mathbf{H 1}, \mathbf{H} 2$ and if $\beta_{0}<2 \min \left(\beta_{\mathrm{L}}, \beta_{\mathrm{R}}\right)$ there is a $\lambda^{*}>0$ such that if the couplings satisfy $\left|\lambda_{\mathrm{L}, m}\right|,\left|\lambda_{\mathrm{R}, m}\right| \in\left(0, \lambda^{*}\right)$ then both $L_{\mathcal{H}_{0}}$ and $L_{\mathcal{H}_{0}}^{*}$ have compact resolvent.

A useful by-product of the proof Proposition 3.4 are some additional smoothness and decay properties of the eigenvalues of $L_{\mathcal{H}_{0}}$ and $L_{\mathcal{H}_{0}}^{*}$ on $\mathcal{H}_{0}$.

Proposition 3.5. Let $g$ denote an eigenvector of $L_{\mathcal{H}_{0}}$ or $L_{\mathcal{H}_{0}}^{*}$. If the assumptions of Proposition 3.4 are satisfied then we have

$$
g e^{-\beta_{0} G / 2} \in \mathcal{S}(X),
$$

where $\mathcal{S}(X)$ denotes the Schwartz space.

Using these results, we come back to the Markov process defined by the Eqs.(2.9), and whose semi-group $T^{t}$ was defined in Eq.(3.6). We prove the existence of an invariant measure with a smooth density and give a bound which shows that, in some sense, the chain does not get hotter than the hottest heat bath.

Proposition 3.6. Under the assumptions H1-H2 there is a $\lambda^{*}>0$ such that if the couplings satisfy $\left|\lambda_{\mathrm{L}, m}\right|,\left|\lambda_{\mathrm{R}, m}\right| \in\left(0, \lambda^{*}\right)$ the Markov process $T^{t}$ has an invariant measure $\mu$ which is 
absolutely continuous with respect to the Lebesgue measure. Its density $h$ satisfies the following: $h \exp (\beta G) \in \mathcal{S}(X)$ for all $\beta<\min \left(\beta_{\mathrm{L}}, \beta_{\mathrm{R}}\right)$.

Proof. The function 1 is obviously a solution of $L f=0$ with $L$ defined in Eq.(3.11). Note next that the function 1 is in $\mathcal{H}_{0}$, as is seen from Eq.(3.9) (if $\left|\lambda_{\mathrm{L}, m}\right|$ and $\left|\lambda_{\mathrm{R}, m}\right|$ are sufficiently small). Since, by Proposition 3.4, the operator $L_{\mathcal{H}_{0}}$ has compact resolvent on $\mathcal{H}_{0}$, it follows that 0 is also an eigenvalue of $L_{\mathcal{H}_{0}}^{*}$. Let us denote the corresponding eigenvector by $g$. We will choose the normalization $(g, 1)_{\mathcal{H}_{0}}=1$. We assume first that $g \geq 0$. Then the function

$$
h(x)=Z_{0}^{-1} g(x) e^{-\beta_{0} G(x)},
$$

with $\beta_{0}$ and $G$ defined in Eqs.(3.8) and (3.7), is the density of an invariant measure for the process $T^{t}$ : Indeed, we note first that $\|h\|_{\mathrm{L}^{1}(X, \mathrm{dx})}=(1, g)_{\mathcal{H}_{0}}$ is finite and thus $\mu(\mathrm{dx})$ is a probability measure.

Let $E$ be some Borel set. Then the characteristic function $\chi_{E}$ of $E$ belongs to $\mathcal{H}_{0}$. We have

$$
\begin{aligned}
\int \mu(\mathrm{dx}) T^{t} \chi_{E} & =Z_{0}^{-1} \int \mathrm{dx} e^{-\beta_{0} G(x)} g(x) T^{t} \chi_{E} \\
& =Z_{0}^{-1} \int \mathrm{dx} e^{-\beta_{0} G(x)}\left(T_{\mathcal{H}_{0}}^{t}\right)^{*} g(x) \chi_{E} \\
& =\mu(E),
\end{aligned}
$$

and therefore $\mu(\mathrm{dx})$ is an invariant measure for the Markov process (2.9).

To complete the first part of the proof of Proposition 3.6 it remains to show that $g \geq 0$. We will do this by checking that $h \geq 0$. We need some notation. Let $L^{\mathrm{T}}$ denote the formal adjoint of $L$. Then one has $L^{\mathrm{T}} h=0$. This follows from the identities

$$
\begin{aligned}
\int \mathrm{dx} f L^{\mathrm{T}} h & =Z_{0}^{-1} \int \mathrm{dx} f L^{\mathrm{T}}\left(g e^{-\beta_{0} G}\right) \\
& =Z_{0}^{-1} \int \mathrm{dx}(L f) g e^{-\beta_{0} G} \\
& =(L f, g)_{\mathcal{H}_{0}}=\left(f, L_{\mathcal{H}_{0}}^{*} g\right)_{\mathcal{H}_{0}}=(f, 0)_{\mathcal{H}_{0}}=0,
\end{aligned}
$$

which hold for all $f \in \mathcal{C}_{0}^{\infty}(X)$. Consider now the semi-group $T^{t}$ acting on the space $\mathcal{C}_{\infty}(X)$ defined at the beginning of Section 3. Since $T^{t}$ is a Markovian semi-group, we have $T^{t} 1=1$ and $T^{t}$ is positivity preserving. The operator $T^{t}$ induces an action $\left(T^{t}\right)^{*}$ defined on the dual space $\mathcal{C}_{\infty}^{*}(X)$ which consists of finite measures. Since $T^{t}$ is Markovian, $\left(T^{t}\right)^{*}$ maps probability measures to probability measures. Furthermore, if a measure $\nu$ has a density $f$ in $\mathrm{L}^{1}(X, \mathrm{dx})$, then $\left(T^{t}\right)^{*} \nu$ is a measure which has again a density in $\mathrm{L}^{1}(X, \mathrm{dx})$ : Indeed, by Corollary 3.3 the transition probabilities of the Markov process $P(t, x, y)$ are in $\mathcal{C}^{\infty}((0, \infty) \times X \times X)$. If we denote by $\left(T^{t}\right)^{\mathrm{T}}$ the induced action of $\left(T^{t}\right)^{*}$ on the densities, we have for $g \geq 0$,

$$
\left(T^{t}\right)^{*}(g(x) \mathrm{dx})=\int \mathrm{dy} g(y) P(t, y, \mathrm{dx})=\mathrm{dx} \int \mathrm{dy} g(y) P(t, y, x)=\left(\left(T^{t}\right)^{\mathrm{T}} g\right)(x) \mathrm{dx},
$$


and $\left\|\left(T^{t}\right)^{\mathrm{T}} g\right\|_{\mathrm{L}^{1}}=\|g\|_{\mathrm{L}^{1}}$.

Coming back to the invariant density $h$, we know that

$$
\left(T^{t}\right)^{\mathrm{T}} h=h .
$$

We next show $\left(T^{t}\right)^{\mathrm{T}}|h|=|h|$. Since $|h| \pm h \geq 0$, we have $\left(T^{t}\right)^{\mathrm{T}}(|h| \pm h) \geq 0$. This can be rewritten as

$$
\left|\left(T^{t}\right)^{\mathrm{T}} h\right| \leq\left(T^{t}\right)^{\mathrm{T}}|h| .
$$

Therefore,

$$
|h|=\left|\left(T^{t}\right)^{\mathrm{T}} h\right| \leq\left(T^{t}\right)^{\mathrm{T}}|h| .
$$

Since $\left(T^{t}\right)^{\mathrm{T}}$ preserves the $\mathrm{L}^{1}$-norm, we conclude that

$$
|h|=\left(T^{t}\right)^{\mathrm{T}}|h| .
$$

This shows the existence of an invariant measure.

Now, by Proposition 3.5, we have $h \exp (\beta G / 2) \in \mathcal{S}(X)$ for all $\beta<2 \min \left(\beta_{\mathrm{L}}, \beta_{\mathrm{R}}\right)$ and so for $\beta<\min \left(\beta_{\mathrm{L}}, \beta_{\mathrm{R}}\right)$ it follows that $h \exp (\beta G) \in \mathcal{S}(X)$. This concludes the proof of Proposition 3.6.

We next prove the uniqueness of the invariant measure and the ergodic properties of the Markov process. We start by fixing an inverse temperature $\beta_{0}$. If $\beta_{\mathrm{L}}=\beta_{\mathrm{R}}=\beta_{0}$, the two heat baths are at the same temperature, and the equilibrium state of the system is known, since it is given by the generalized Gibbs distribution $Z_{0}^{-1} e^{-\beta_{0} G}$. For the equilibrium case, this distribution is the unique invariant measure. The existence is obvious from what we showed for the case of arbitrary temperatures. To show uniqueness, assume that there is a second invariant measure. Since $L^{T}$ is hypoelliptic, then by Corollary 3.2 this measure has a smooth density. Since different smooth invariant measures have mutually disjoint supports and $e^{-\beta_{0} G}$ has support everywhere, uniqueness follows. If the invariant measure is unique, it is ergodic and hence, (see [Yo] and [Ho]) 0 is a simple eigenvalue of $L_{\mathcal{H}_{0}}$.

The case of different temperatures will be handled by a perturbation argument around the equilibrium situation we just described. This perturbation argument will take place in the fixed Hilbert space $\mathcal{H}_{0}$ defined in Eq.(3.9). Thus, we will consider values of $\beta_{\mathrm{L}}$ and $\beta_{\mathrm{R}}$ such that

$$
\frac{1}{\beta_{0}}=\frac{1}{2}\left(\frac{1}{\beta_{\mathrm{L}}}+\frac{1}{\beta_{\mathrm{R}}}\right), \quad \frac{\left|\beta_{\mathrm{L}}-\beta_{\mathrm{R}}\right|}{\beta_{\mathrm{L}}+\beta_{\mathrm{R}}}<\varepsilon,
$$

for some small $\varepsilon>0$ (which does not depend on $\beta_{0}$ ).

We first show that 0 remains a simple eigenvalue of the generator $L_{\mathcal{H}_{0}}$ when the temperature difference satisfies (3.19) for a sufficiently small $\varepsilon$.

Lemma 3.7. Under the assumptions $\mathbf{H 1}-\mathbf{H} 2$ there are constants $\lambda^{*}>0$ and $\varepsilon>0$ such that if the couplings satisfy $\left|\lambda_{\mathrm{L}, m}\right|,\left|\lambda_{\mathrm{R}, m}\right| \in\left(0, \lambda^{*}\right)$ and moreover $\beta_{\mathrm{L}}, \beta_{\mathrm{R}}$ satisfy (3.19), then 0 is a simple eigenvalue of the generator $L_{\mathcal{H}_{0}}$.

Proof. It will be convenient to work in the flat Hilbert space $\mathrm{L}^{2}(X, \mathrm{dx})$. Note that $\mathcal{K}=$ $\exp \left(-\beta_{0} G / 2\right) L \exp \left(+\beta_{0} G / 2\right)$ is a function $\mathcal{K} \equiv \mathcal{K}\left(\beta_{\mathrm{L}}, \beta_{\mathrm{R}}, \beta_{0}\right)$. We write this as

$$
\mathcal{K}\left(\beta_{\mathrm{L}}, \beta_{\mathrm{R}}, \beta_{0}\right)=\mathcal{K}\left(\beta_{0}, \beta_{0}, \beta_{0}\right)+\delta Z,
$$


where

$$
\delta=\frac{\beta_{\mathrm{R}}-\beta_{\mathrm{L}}}{\beta_{\mathrm{R}}+\beta_{\mathrm{L}}}
$$

One finds

$$
\begin{aligned}
\mathcal{K}\left(\beta_{0}, \beta_{0}, \beta_{0}\right) & =\sum_{m=1}^{M} \frac{\lambda_{\mathrm{L}, m}^{2} \gamma_{\mathrm{L}, m}}{2}\left(\frac{2}{\beta_{0}} \nabla_{r_{\mathrm{L}, m}}^{2}-\frac{\beta_{0}}{2} W_{\mathrm{L}, m}^{2}+\frac{d}{\lambda_{\mathrm{L}, m}^{2}}\right) \\
& +\sum_{m=1}^{M} \frac{\lambda_{\mathrm{R}, m}^{2} \gamma_{\mathrm{R}, m}}{2}\left(\frac{2}{\beta_{0}} \nabla_{r_{\mathrm{R}, m}}^{2}-\frac{\beta_{0}}{2} W_{\mathrm{R}, m}^{2}+\frac{d}{\lambda_{\mathrm{R}, m}^{2}}\right) \\
& +\sum_{m=1}^{M} r_{\mathrm{L}, m} \cdot \nabla_{p_{1}}+L_{\mathrm{S}}+\sum_{m=1}^{M} r_{\mathrm{R}, m} \cdot \nabla_{p_{n}},
\end{aligned}
$$

and

$$
\begin{aligned}
Z & =\sum_{m=1}^{M} \frac{\lambda_{\mathrm{L}, m}^{2} \gamma_{\mathrm{L}, m}}{2}\left(\frac{2}{\beta_{0}} \nabla_{r_{\mathrm{L}, m}}^{2}+W_{\mathrm{L}, m} \cdot \nabla_{r_{\mathrm{L}, m}}+\nabla_{r_{\mathrm{L}, m}} \cdot W_{\mathrm{L}, m}+\frac{\beta_{0}}{2} W_{\mathrm{L}, m}^{2}\right) \\
& -\sum_{m=1}^{M} \frac{\lambda_{\mathrm{R}, m}^{2} \gamma_{\mathrm{R}, m}}{2}\left(\frac{2}{\beta_{0}} \nabla_{r_{\mathrm{R}, m}}^{2}+W_{\mathrm{R}, m} \cdot \nabla_{r_{\mathrm{R}, m}}+\nabla_{r_{\mathrm{R}, m}} \cdot W_{\mathrm{R}, m}+\frac{\beta_{0}}{2} W_{\mathrm{R}, m}^{2}\right) .
\end{aligned}
$$

Furthermore, by Proposition 3.4, $R_{0} \equiv\left(1-\mathcal{K}\left(\beta_{0}, \beta_{0}, \beta_{0}\right)\right)^{-1}$ is a compact operator, and therefore the simple eigenvalue 1 of $R_{0}$ is isolated. From now on we assume for convenience that $\alpha \equiv \alpha\left(\beta_{\mathrm{L}}, \beta_{\mathrm{R}}\right)$ is strictly smaller than one. Note that this is no restriction of generality: if $\alpha \in[n-1, n)$ with $n>1$, we replace $(1-\mathcal{K})^{-1}$ by $\left(1-\frac{1}{n} \mathcal{K}\right)^{-1}$ in the following discussion.

We show next that the resolvent $R\left(\beta_{\mathrm{L}}, \beta_{\mathrm{R}}, \beta_{0}\right) \equiv\left(1-\mathcal{K}\left(\beta_{\mathrm{L}}, \beta_{\mathrm{R}}, \beta_{0}\right)\right)^{-1}$ depends analytically on the parameter $\delta$. It is convenient to write the perturbation $Z$ as

$$
Z=\sum_{j=1}^{N} E_{j} F_{j},
$$

where the $E_{j}$ and $F_{j}$ are of the form const. $\partial_{r_{i, m}^{(\nu)}}$ or const. $W_{i, m}^{(\nu)}, i \in\{\mathrm{L}, \mathrm{R}\}, m=1, \ldots, M$, and $N=8 d M$. With the matrix notation

$$
F=\left(\begin{array}{c}
F_{1} \\
\vdots \\
F_{N}
\end{array}\right), \quad E^{T}=\left(E_{1}, \ldots, E_{N}\right)
$$

we can write $Z$ as $Z=E^{T} F$. We will use the following resolvent formula

$$
R\left(\beta_{\mathrm{L}}, \beta_{\mathrm{R}}, \beta_{0}\right)=R_{0}\left(1+\delta R_{0} E^{T}\left(1-\delta F R_{0} E^{T}\right)^{-1} F R_{0}\right) .
$$


To justify Eq.(3.20) we have to show that for $\delta$ small enough the operator-valued matrix $\left(1-\delta F R_{0} E^{T}\right)$ is invertible. It is enough to show that $F_{j} R_{0} E_{k}$ is a bounded operator, for all $j, k$. For this we decompose $1-\mathcal{K}\left(\beta_{0}, \beta_{0}, \beta_{0}\right)$ into its symmetric and antisymmetric parts:

$$
1-\mathcal{K}\left(\beta_{0}, \beta_{0}, \beta_{0}\right)=X+i Y
$$

where

$$
\begin{aligned}
X=1 & +\sum_{m=1}^{M} \frac{\lambda_{\mathrm{L}, m}^{2} \gamma_{\mathrm{L}, m}}{2}\left(-\frac{2}{\beta_{0}} \nabla_{r_{\mathrm{L}, m}}^{2}+\frac{\beta_{0}}{2} W_{\mathrm{L}, m}^{2}-d \frac{1}{\lambda_{\mathrm{L}, m}^{2}}\right) \\
& +\sum_{m=1}^{M} \frac{\lambda_{\mathrm{R}, m}^{2} \gamma_{\mathrm{R}, m}}{2}\left(-\frac{2}{\beta_{0}} \nabla_{r_{\mathrm{R}, m}}^{2}+\frac{\beta_{0}}{2} W_{\mathrm{R}, m}^{2}-d \frac{1}{\lambda_{\mathrm{R}, m}^{2}}\right) .
\end{aligned}
$$

From the simple estimates

$$
\begin{aligned}
\left\|E_{j} f\right\|^{2} & \leq(f, X f), \\
\left\|F_{j} f\right\|^{2} & \leq(f, X f),
\end{aligned}
$$

which hold for $f \in \mathcal{C}_{0}^{\infty}(X)$, and since $X$ is a strictly positive operator we see that $E_{j} X^{-1 / 2}$ and $F_{j} X^{-1 / 2}$ are bounded operators. From the identity

$$
\begin{aligned}
E_{j} R_{0} F_{k} & =E_{j}(X+i Y)^{-1} F_{k} \\
& =E_{j} X^{-1 / 2}\left(1+i X^{-1 / 2} Y X^{-1 / 2}\right)^{-1} X^{-1 / 2} F_{k},
\end{aligned}
$$

we see that $E_{j} R_{0} F_{k}$ are bounded operators for all $j, k$. Therefore the r.h.s of Eq.(3.20) is well defined for sufficiently small $\delta$. An immediate consequence of the resolvent formula (3.20) is that for sufficiently small $\delta$ the spectrum of $R\left(\beta_{\mathrm{L}}, \beta_{\mathrm{R}}, \beta_{0}\right)$ has the same form as the spectrum $R_{0}$ : 1 is an eigenvalue and there is a spectral gap and, in particular 1 is a simple eigenvalue. This concludes the proof of Lemma 3.7.

Next we use this lemma to prove uniqueness of the invariant measure. We have the following

Theorem 3.8. Under the assumptions $\mathbf{H 1}-\mathbf{H} 2$ there are constants $\lambda^{*}>0$ and $\varepsilon>0$ such that if the couplings satisfy $\left|\lambda_{\mathrm{L}, m}\right|,\left|\lambda_{\mathrm{R}, m}\right| \in\left(0, \lambda^{*}\right)$ and the temperatures satisfy $\left|\beta_{\mathrm{R}}-\beta_{\mathrm{L}}\right| /\left(\beta_{\mathrm{L}}+\right.$ $\beta_{\mathrm{R}}$ ) $<\varepsilon$, the Markov process $T^{t}$ has a unique (and hence ergodic) invariant measure.

Proof. The proof uses a dynamical argument. By Proposition 3.4 we have in the Hilbert space $\mathcal{H}_{0}$ (with $\beta_{0}$ given as in (3.19)) the eigenvalue equation $T_{\mathcal{H}_{0}}^{t} 1=1$ and $\left(T_{\mathcal{H}_{0}}^{t}\right)^{*} g=g$. Let the eigenvectors be normalized such that $(g, 1)_{\mathcal{H}_{0}}=1$. By Lemma 3.7, 0 is a simple eigenvalue of the generator $L_{\mathcal{H}_{0}}$ if $\left(\beta_{\mathrm{R}}-\beta_{\mathrm{L}}\right) /\left(\beta_{\mathrm{L}}+\beta_{\mathrm{R}}\right)$ is small enough and by Proposition 3.6, the measure $\mu(\mathrm{dx})=Z_{0}^{-1} g \exp \left(-\beta_{0} G\right)$ is an invariant measure for the Markov process. It is absolutely continuous with respect to the Lebesgue measure which we denote by $\lambda$.

Assume now that $\nu$ is another invariant probability measure. By the hypoellipticity of $L$ it must have a smooth density. Therefore there is a Borel set $A \subset X$, which we may assume 
bounded, with the following properties: we have $\nu(A)>0$ and $\lambda(A)>0$ but $\mu(A)=0$, because the measures have disjoint supports. Let $\chi_{A}$ denote the characteristic function of the set $A$. By the pointwise ergodic theorem, see [Yo] and [Ho], we have, denoting $\sigma_{s}(x)$ the ergodic average $\sigma_{s}(x)=(1 / s) \int_{0}^{s} \mathrm{~d} t T^{t} \chi_{A}(x)$

$$
\lim _{s \rightarrow \infty} \sigma_{s}(x)=\nu(A), \quad \nu \text {-a.e. } .
$$

Since $T^{t}$ is a contraction semi-group on $B(X, \mathcal{B})$, and $\chi_{A} \leq 1$ we find $\left\|\sigma_{s}\right\|_{\infty} \leq 1$, for all $s>0$. From the easy bound

$$
\left\|\sigma_{s}\right\|_{\mathcal{H}_{0}} \leq\left\|\sigma_{s}\right\|_{\infty},
$$

we see that the set $\left\{\sigma_{s}, s>0\right\}$ is a bounded subset of $\mathcal{H}_{0}$ and hence weakly sequentially precompact. Therefore there is a sequence $s_{n} \uparrow \infty$ such that

$$
\underset{n \rightarrow \infty}{\mathrm{w}-\lim } \sigma_{s_{n}}=\sigma^{*}
$$

where $\mathrm{w}-\lim$ denotes the weak limit in $\mathcal{H}_{0}$. Since $T^{u}$ is a bounded operator for all $u>0$, we have

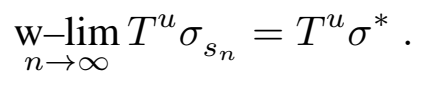

We next show

$$
T^{u} \sigma^{*}=\sigma^{*} .
$$

Indeed,

$$
\begin{aligned}
T^{u} \sigma_{s_{n}}(x) & =\frac{1}{s_{n}} \int_{u}^{s_{n}+u} \mathrm{~d} t T^{t} \chi_{A}(x) \\
& =\sigma_{s_{n}}-\frac{1}{s_{n}} \int_{0}^{u} \mathrm{~d} t T^{t} \chi_{A}(x)+\frac{1}{s_{n}} \int_{s_{n}}^{s_{n}+u} \mathrm{~d} t T^{t} \chi_{A}(x) .
\end{aligned}
$$

The last two terms in (3.23) are bounded by $u / s_{n}$ and we obtain $T^{u} \sigma^{*}=\sigma^{*}$ for all $u>0$ by taking the limit $n \rightarrow \infty$ in (3.23).

Therefore, $\sigma^{*}$ is in the eigenspace of the eigenvalue 1 of $T_{\mathcal{H}_{0}}^{t}, t>0$ and so, by Lemma 3.7 $\sigma^{*}=c 1$. To compute $c$ we note that $c=\left(g, \sigma^{*}\right)_{\mathcal{H}_{0}}$ and, using the invariance of the measure, we get

$$
\begin{aligned}
c & =\lim _{n \rightarrow \infty}\left(g, \frac{1}{s_{n}} \int_{0}^{s_{n}} \mathrm{~d} t T_{\mathcal{H}_{0}}^{t} \chi_{A}\right)_{\mathcal{H}_{0}} \\
& =\lim _{n \rightarrow \infty} \frac{1}{s_{n}} \int_{0}^{s_{n}} \mathrm{~d} t \int \mu(\mathrm{dx}) T^{t} \chi_{A}=\mu(A) .
\end{aligned}
$$

So we have $c=\mu(A)$ and $\mu(A)=0$ by hypothesis. Using this information, we consider $\left(\chi_{A}, \sigma_{s_{n}}\right)_{\mathcal{H}_{0}}$. We have, on one hand,

$$
\lim _{n \rightarrow \infty}\left(\chi_{A}, \sigma_{s_{n}}\right)_{\mathcal{H}_{0}}=\left(\chi_{A}, \sigma^{*}\right)_{\mathcal{H}_{0}}=0,
$$


and on the other hand we have, by Eq.(3.21) and by the dominated convergence theorem,

$$
\lim _{n \rightarrow \infty}\left(\chi_{A}, \sigma_{s_{n}}\right)_{\mathcal{H}_{0}}=\int \mathrm{dx} \nu(A) \chi_{A} Z_{0}^{-1} e^{-\beta_{0} G}>0
$$

and this is a contradiction. This shows that there is a unique invariant measure for the Markov process $T^{t}$ and as a consequence the measure is ergodic.

We will now strengthen the last statement by showing that the invariant measure is in fact mixing. This will be done by extending the proof of return to equilibrium given in [Tr].

Proposition 3.9. Assume that the conditions of Theorem 3.8 are satisfied. Then the invariant measure $\mu(\mathrm{dx})$ for the Markov process $T^{t}$ is mixing, i.e.,

$$
\lim _{t \rightarrow \infty} \int \mu(\mathrm{dx}) f(x) T^{t} g(x)=\int \mu(\mathrm{dx}) f(x) \int \mu(\mathrm{dx}) g(x)
$$

for all $f, g \in \mathrm{L}^{2}(X, \mu(\mathrm{dx}))$.

Proof. We denote $\mathcal{H}=\mathrm{L}^{2}(X, \mu(\mathrm{dx}))$ and its scalar product by $(\cdot, \cdot)_{\mathcal{H}}$ and by $\|\cdot\|_{\mathcal{H}}$ its norm. By [Yo], Chap XIII.1, Thm 1, $T^{t}$ defines a contraction semi-group on $\mathcal{H}$. Since $T^{t}$ is a strongly continuous semi-group on $\mathcal{C}_{\infty}(X)$ (see $[\mathrm{Ne}]$ ) and since $\mathcal{C}_{\infty}(X)$ is dense in $\mathcal{H}$, we can extend $T^{t}$ to a strongly continuous semi-group $T_{\mathcal{H}}^{t}$ on $\mathcal{H}$. The property of mixing is equivalent to

$$
\underset{t \rightarrow \infty}{\mathrm{w}-\lim _{\mathcal{H}}} T_{\mathcal{H}}^{t} f=(1, f)_{\mathcal{H}} \quad \text { for all } f \in \mathcal{H}
$$

By a simple density argument it is enough to show (3.24) for a dense subset of $\mathcal{H}$. Let $\mathcal{C}^{2}(X)$ denote the bounded continuous function whose first and second partial derivatives are bounded and continuous. Then- [GS], Part II, $\S 9$ - if $f \in \mathcal{C}^{2}(X)$, then $T_{\mathcal{H}}^{t} f \in \mathcal{C}^{2}(X)$ and for any $\tau<\infty, T_{\mathcal{H}}^{t} f$ is uniformly differentiable w.r.t. $t \in[0, \tau]$ and

$$
\frac{\partial}{\partial t} T_{\mathcal{H}}^{t} f=L f
$$

where $L$ is the differential operator given in (3.11). Let $f \in \mathcal{C}^{2}(X)$. Using the fact, see Proposition 3.5 and Proposition 3.6, that the density of the invariant measure is of the form $h(x)=\tilde{g} e^{-\beta_{0} G / 2}$ with $g \in \mathcal{S}(X)$, we may differentiate under the integral and integrate by parts and using the invariance of the measure we obtain

$$
\frac{\mathrm{d}}{\mathrm{d} t}\left\|T_{\mathcal{H}}^{t} f\right\|_{\mathcal{H}}^{2}=\left(L T_{\mathcal{H}}^{t} f, T_{\mathcal{H}}^{t} f\right)_{\mathcal{H}}+\left(T_{\mathcal{H}}^{t} f, L T_{\mathcal{H}}^{t} f\right)_{\mathcal{H}}=-\sum_{\substack{i \in\{\mathrm{L}, \mathrm{R}\} \\ m \in\{1, \ldots, M\} \\ \nu \in\{1, \ldots, d\}}} \frac{2 \lambda_{i, m}^{2} \gamma_{i, m}}{\beta_{i}}\left\|\partial_{r_{i, m}^{(\nu)}} T_{\mathcal{H}}^{t} f\right\|_{\mathcal{H}}^{2}
$$


where $\partial_{r_{i, m}^{(\nu)}}$ is the differential operator with domain $\mathcal{C}^{2}(X)$. Thus $\left\|T_{\mathcal{H}}^{t} f\right\|_{\mathcal{H}}^{2}$ is decreasing, bounded below and continuous and so $\lim _{t \rightarrow \infty}\left\|T_{\mathcal{H}}^{t} f\right\|_{\mathcal{H}}^{2}$ exists. As a consequence we find

$$
\left\|\partial_{r_{i, m}^{(\nu)}} T_{\mathcal{H}}^{t} f\right\|_{\mathcal{H}}^{2} \in \mathrm{L}^{1}([0, \infty), d t)
$$

Following [Tr] and [Br], we call a sequence $\left\{t_{n}\right\}$ a $(*)$-sequence if $t_{n} \uparrow \infty$ and

$$
\lim _{n \rightarrow \infty}\left\|\partial_{r_{i, m}^{(\nu)}} T_{\mathcal{H}}^{t_{n}} f\right\|_{\mathcal{H}}^{2}=0
$$

The existence of $(*)$-sequences for our problem follows easily from (3.26). Further we define an almost $(*)$-sequence as a sequence $s_{n} \uparrow \infty$ for which there exists a $(*)$-sequence $\left\{t_{n}\right\}$ with $\left|s_{n}-t_{n}\right| \rightarrow 0$ as $n \rightarrow \infty$. As in [Tr] we next show that w- $\lim _{n \rightarrow \infty} T_{\mathcal{H}}^{t_{n}} f=\mathrm{w}-\lim _{n \rightarrow \infty} T_{\mathcal{H}}^{s_{n}} f$. Indeed, let us choose $\tau<\min \left(t_{1}, s_{1}\right)$. From the inequality

$$
\left\|\frac{\mathrm{d}}{\mathrm{d} t} T_{\mathcal{H}}^{t} f\right\|_{\mathcal{H}}=\left\|T_{\mathcal{H}}^{t-\tau} L T_{\mathcal{H}}^{\tau} f\right\|_{\mathcal{H}} \leq\left\|L T_{\mathcal{H}}^{\tau} f\right\|_{\mathcal{H}}
$$

which holds for $t>\tau$, we have

$$
\left\|\left(T_{\mathcal{H}}^{s_{n}}-T_{\mathcal{H}}^{t_{n}}\right) f\right\|_{\mathcal{H}} \leq\left|s_{n}-t_{n}\right|\left\|L T_{\mathcal{H}}^{\tau} f\right\|_{\mathcal{H}} \rightarrow 0, \quad n \rightarrow \infty
$$

which shows that $T_{\mathcal{H}}^{t_{n}} f$ and $T_{\mathcal{H}}^{s_{n}} f$ have the same weak limit.

The set $\left\{T_{\mathcal{H}}^{t} f, t \geq 0\right\}$ is bounded and hence sequentially weakly precompact, so that by passing to a subsequence, we may assume that

$$
\underset{n \rightarrow \infty}{\mathrm{w}-\lim _{\mathcal{H}}} T_{\mathcal{H}}^{t_{n}} f=\gamma, \quad \gamma \in \mathcal{H}
$$

Next we show that for all $(*)$-sequences $\mathrm{w}-\lim _{n \rightarrow \infty} T_{\mathcal{H}}^{t_{n}}$ is a locally constant function $\mu-a . e$. We begin by showing that $\gamma$ does not depend on the variables $r$.

Let $\partial_{r_{i, m}^{(\nu)}}^{*}$ denote the adjoint of $\partial_{r_{i, m}^{(\nu)}}$ in $\mathcal{H}$ and let $\psi \in \mathcal{C}_{0}^{\infty}(X)$. By the smoothness properties of the density of the invariant measure we see that the function $\psi$ is in the domain of $\partial_{r_{i, m}^{(\nu)}}^{*}$ and we have, using (3.27)

$$
\left(\gamma, \partial_{r_{i, m}^{(\nu)}}^{*} \psi\right)_{\mathcal{H}}=\lim _{n \rightarrow \infty}\left(T_{\mathcal{H}}^{t_{n}} f, \partial_{r_{i, m}^{(\nu)}}^{*} \psi\right)_{\mathcal{H}}=\lim _{n \rightarrow \infty}\left(\partial_{r_{i, m}^{(\nu)}} T_{\mathcal{H}}^{t_{n}} f, \psi\right)_{\mathcal{H}}=0
$$

Written explicitly,

$$
\int \operatorname{dp} \operatorname{dq} \operatorname{dr} \gamma(p, q, r) \partial_{r_{i, m}^{(\nu)}}(\psi(p, q, r) h(p, q, r))=0,
$$

for any $\psi \in \mathcal{C}_{0}^{\infty}(X)$. Since $\gamma \in \mathcal{H}$, we may set $\gamma=0$ on the set $A \equiv\{x \in X ; h(x)=0\}$ and $\gamma$ is locally integrable and thus defines a distribution in $\mathcal{D}^{\prime}(X)$. By Eq.(3.28) the support of the 
distribution $\partial_{r_{i, m}^{(\nu)}} \gamma(p, q, r)$ does not intersect the set $A$ and thus $\gamma(p, q, r)$ is $\mu$-a.e. independent of $r$.

Let $t>0$. Then $\mathrm{w}-\lim _{n \rightarrow \infty} T_{\mathcal{H}}^{t_{n}+t} f=T_{\mathcal{H}}^{t} \gamma$. Since $t+t_{n} \uparrow \infty$, it is easy to show, see [Br], that $t_{n}+t$ has an almost $(*)$-subsequence $s_{n}$ and from the above arguments we conclude that $T_{\mathcal{H}}^{t} \gamma$ is independent of $r$.

Next we show inductively, using Condition $\mathbf{H 2}$ that $\gamma$ does not depend on the variables $p$, $q$. Let $\left(T_{\mathcal{H}}^{t}\right)^{*}$ denote the semi-group dual to $T_{\mathcal{H}}^{t}$ on $\mathcal{H}$ and denote $Z$ its generator.

Note that for $\psi \in \mathcal{C}_{0}^{\infty}(X)$ we have, upon integrating by parts

$$
\begin{aligned}
\frac{\mathrm{d}}{\mathrm{d} t}\left(\psi, T_{\mathcal{H}}^{t} f\right)_{\mathcal{H}} & =\left(\psi, L T_{\mathcal{H}}^{t} f\right)_{\mathcal{H}} \\
& =\int \mathrm{dpdqdr} \sum_{\substack{i \in\{\mathrm{L}, \mathrm{R}\} \\
m \in\{1, \ldots, M\}}} \frac{\lambda_{i, m}^{2} \gamma_{i, m}}{\beta_{i}}\left[\nabla_{r_{i, m}} \cdot\left(\nabla_{r_{i, m}}+\beta_{i} W_{i, m}\right) \bar{\psi} h\right] T_{\mathcal{H}}^{t} f \\
& -\int \mathrm{dpdqdr}\left(L_{0}(\bar{\psi} h)\right) T_{\mathcal{H}}^{t} f,
\end{aligned}
$$

where $L_{0}$ is given by

$$
L_{0}=\sum_{m=1}^{M} r_{\mathrm{L}, m} \cdot \nabla_{p_{1}}+\sum_{m=1}^{M} r_{\mathrm{R}, m} \cdot \nabla_{p_{n}}+\sum_{j=1}^{n} p_{j} \cdot \nabla_{q_{j}}-\left(\nabla_{q_{j}} V\right) \cdot \nabla_{p_{j}} .
$$

Since $\mathcal{C}_{0}^{\infty}(X)$ is in the domain of $Z$, we get

$$
\begin{gathered}
\frac{\mathrm{d}}{\mathrm{d} t}\left(\psi, T_{\mathcal{H}}^{t} \gamma\right)_{\mathcal{H}}=\left(Z \psi, T_{\mathcal{H}}^{t} \gamma\right)_{\mathcal{H}}=\lim _{n \rightarrow \infty}\left(Z \psi, L T_{\mathcal{H}}^{t_{n}+t} f\right)_{\mathcal{H}} \\
=\left(Z \psi, T_{\mathcal{H}}^{t} \gamma\right)_{\mathcal{H}}=-\int \mathrm{dp} \operatorname{dq} \operatorname{dr}\left(L_{0}(\bar{\psi} h)\right) T_{\mathcal{H}}^{t} \gamma .
\end{gathered}
$$

The last equality follows from (3.29) since $T_{\mathcal{H}}^{t} \gamma$ is independent of $r$.

We next choose $\psi(p, q, r) \in \mathcal{C}_{0}^{\infty}(X)$ of the form $\psi(r, p, q)=\varphi_{1}(r) \varphi_{2}(p, q) h^{-1}(p, q, r)$ with $\operatorname{supp}\left(\varphi_{1}(r) \varphi_{2}(p, q)\right) \cap A=\emptyset$ and $\int \operatorname{dr} \varphi_{1}(r)=0$. For this choice of $\psi$ we have

$$
\left(\psi, T_{\mathcal{H}}^{t} \gamma\right)_{\mathcal{H}}=\int \mathrm{dpdq}\left(T_{\mathcal{H}}^{t} \gamma\right)(p, q) \bar{\varphi}_{2}(p, q) \cdot \int \operatorname{dr} \bar{\varphi}_{1}(r)=0,
$$

and therefore

$$
\begin{aligned}
0 & =\int \mathrm{dpdq} \operatorname{dr} \gamma(p, q) L_{0}\left(\bar{\varphi}_{1}(r) \bar{\varphi}_{2}(p, q)\right) \\
& =\int \mathrm{dpdq} \gamma(p, q) \nabla_{p_{1}} \bar{\varphi}_{2}(p, q) \cdot \int \mathrm{dr} \sum_{m=1}^{M} r_{\mathrm{L}, m} \bar{\varphi}_{1}(r) \\
& +\int \mathrm{dpdq} \gamma(p, q) \nabla_{p_{n}} \bar{\varphi}_{2}(p, q) \cdot \int \mathrm{dr} \sum_{m=1}^{M} r_{\mathrm{R}, m} \bar{\varphi}_{1}(r) .
\end{aligned}
$$


Since $\varphi_{1}(r)$ is arbitrary, it follows that

$$
\int \mathrm{dpdq} \gamma(p, q) \nabla_{p_{1}} \bar{\varphi}_{2}(p, q)=\int \mathrm{dpdq} \gamma(p, q) \nabla_{p_{n}} \bar{\varphi}_{2}(p, q)=0,
$$

and thus, by a similar argument as above, $\gamma(p, q)$ must be $\mu$-a.e. independent of $p_{1}$ and $p_{n}$ : Thus $\gamma$ is a function $\gamma\left(p_{2}, \ldots, p_{n-1}\right)$.

Using this information, we choose now

$$
\psi(p, q, r)=\varphi_{1}\left(p_{1}, p_{n}\right) \varphi_{2}\left(p_{2}, \ldots, p_{n-1}, q\right) \varphi_{3}(r) h^{-1}(p, q, r),
$$

with $\operatorname{supp}\left(\varphi_{1} \varphi_{2} \varphi_{3}\right) \cap A=\emptyset$ and $\int \operatorname{dp}_{1} \operatorname{dp}_{n} \varphi_{1}\left(p_{1}, p_{n}\right)=0$. For such a choice of $\psi$ we obtain

$$
\begin{aligned}
0 & =\int \mathrm{dpdq} \operatorname{dr} L_{0}\left(\bar{\varphi}_{1} \bar{\varphi}_{2} \bar{\varphi}_{3}\right) \gamma \\
& =\int \mathrm{dp}_{2} \cdots \mathrm{dp}_{n-1} \operatorname{dq} \gamma\left(\nabla_{q_{1}} \bar{\varphi}_{2}\right) \cdot \int \mathrm{dp}_{1} \mathrm{dp}_{n} p_{1} \bar{\varphi}_{1} \int \mathrm{dr} \bar{\varphi}_{3} \\
& +\int \mathrm{dp}_{2} \cdots \mathrm{dp}_{n-1} \operatorname{dq} \gamma\left(\nabla_{q_{n}} \bar{\varphi}_{2}\right) \cdot \int \mathrm{dp}_{1} \mathrm{dp}_{n} p_{n} \bar{\varphi}_{1} \int \mathrm{dr} \bar{\varphi}_{3},
\end{aligned}
$$

and from the arbitrariness of $\varphi_{1}, \varphi_{2}, \varphi_{3}$ we conclude that $\gamma$ is independent of $q_{1}, q_{n}$ (all our statements hold $\mu$-a.e.). Finally, choose

$$
\psi(p, q, r)=\varphi_{1}\left(q_{1}, q_{n}\right) \varphi_{2}\left(p_{2}, \ldots, p_{n-1}, q_{2}, \ldots, q_{n-1}\right) \varphi_{3}\left(p_{1}, p_{n}, r\right) h^{-1}(p, q, r),
$$

with $\operatorname{supp}\left(\varphi_{1} \varphi_{2} \varphi_{3}\right) \cap A=\emptyset$ and $\int \mathrm{dq}_{1} \mathrm{dq}_{n} \varphi_{1}\left(q_{1}, q_{n}\right)=0$. Then we obtain

$$
\begin{aligned}
0 & =\int \mathrm{dp}_{2} \ldots \mathrm{dp}_{n-1} \mathrm{dq}_{2} \ldots \mathrm{dq}_{n-1} \gamma\left(\nabla_{p_{2}} \bar{\varphi}_{2}\right) \cdot \int \mathrm{dq}_{1} \mathrm{dq}_{n}\left(\nabla_{q_{2}} V\right) \bar{\varphi}_{1} \int \mathrm{dp}_{1} \mathrm{dp}_{n} \mathrm{dr} \bar{\varphi}_{3} \\
& +\int \mathrm{dp}_{2} \ldots \mathrm{dp}_{n-1} \mathrm{dq}_{2} \ldots \mathrm{dq}_{n-1} \gamma\left(\nabla_{p_{n-1}} \bar{\varphi}_{2}\right) \cdot \int \mathrm{dq}_{1} \mathrm{dq}_{n}\left(\nabla_{q_{n-1}} V\right) \bar{\varphi}_{1} \int \mathrm{dp}_{1} \mathrm{dp}_{n} \mathrm{dr} \bar{\varphi}_{3} .
\end{aligned}
$$

From the arbitrariness of the $\varphi_{i}$ we conclude in particular that

$$
0=\int \mathrm{dp}_{2} \ldots \mathrm{dp}_{n-1} \mathrm{dq}_{2} \ldots \mathrm{dq}_{n-1} \gamma\left(\nabla_{p_{2}} \bar{\varphi}_{2}\right) \cdot \int \mathrm{dq}_{1} \mathrm{dq}_{n}\left(\nabla_{q_{2}} V\right) \bar{\varphi}_{1} .
$$

We may choose $\varphi_{1}\left(q_{1}, q_{n}\right)=\partial_{q_{1}^{\left(\nu^{\prime}\right)}} \widetilde{\varphi}\left(q_{1}, q_{n}\right)$ for some $\nu^{\prime} \in\{1, \ldots, d\}$ and a positive $\widetilde{\varphi}\left(q_{1}, q_{n}\right)$. By Condition $\mathrm{H} 2$ we see that

$$
X^{\nu, \nu^{\prime}}\left(q_{2}\right) \equiv \int \mathrm{dq}_{1} \mathrm{dq}_{n}\left(\partial_{q_{2}^{(\nu)}} V\right) \varphi_{1}\left(q_{1}, q_{n}\right)=-\int \mathrm{dq}_{1} \mathrm{dq}_{n}\left(\partial_{q_{1}^{\left(\nu^{\prime}\right)}} \partial_{q_{2}^{(\nu)}} V\right) \widetilde{\varphi}_{1}\left(q_{1}, q_{n}\right)
$$

is uniformly positive or negative. We can rewrite (3.30) as

$$
0=\sum_{\nu \in\{1, \ldots, d\}} \int \mathrm{dp}_{2} \ldots \mathrm{dp}_{n-1} \mathrm{dq}_{2} \ldots \mathrm{dq}_{n-1} \gamma \partial_{p_{2}^{(\nu)}} X^{\nu, \nu^{\prime}}\left(q_{2}\right) \bar{\varphi}_{2}
$$


and we conclude that $\gamma$ is independent of $p_{2}$. A similar argument shows that $\gamma$ is independent of $q_{n-1}$ and iterating the above procedure we conclude that $\gamma$ is locally constant $\mu$-a.e.

So far, we have shown that for all $(*)$-sequences $\left\{t_{n}\right\}$ one has $\mathrm{w}-\lim _{n \rightarrow \infty} T_{\mathcal{H}}^{t_{n}} f=\gamma=$ const. From the invariance of the measure $\mu$ and its ergodicity we conclude that

$$
\gamma=(1, f)_{\mathcal{H}}=\int \mu(\mathrm{dx}) f(x) .
$$

We conclude as in [Tr]: suppose that $\mathrm{w}-\lim _{t \rightarrow \infty} T_{\mathcal{H}}^{t} f \neq(1, f)_{\mathcal{H}}$. Then by the weak sequential precompactness of $\left\{T_{\mathcal{H}}^{t} f ; t \geq 0\right\}$, there exists a sequence $u_{n} \uparrow \infty$ for which $\mathrm{w}-\lim _{t \rightarrow \infty} T_{\mathcal{H}}^{t} f=$ $\eta \neq(1, f)_{\mathcal{H}}$. But, referring again to $[\mathrm{Br}]$, the sequence $\left\{u_{n}\right\}$ has an almost $(*)$-subsequence $\left\{s_{n}\right\}$. This implies that there is a $(*)$-sequence $\left\{t_{n}\right\}$ such that $\mathrm{w}-\lim _{n \rightarrow \infty} T_{\mathcal{H}}^{t_{n}} f=\eta$. This is a contradiction, since we have seen that $\mathrm{w}-\lim _{n \rightarrow \infty} T_{\mathcal{H}}^{t_{n}} f=(1, f)_{\mathcal{H}}$ for all $(*)$-sequences. By a simple density argument this implies that

$$
\lim _{t \rightarrow \infty} \int \mu(\mathrm{dx}) f(x) T_{\mathcal{H}}^{t} g(x)=\int \mu(\mathrm{dx}) f(x) \int \mu(\mathrm{dx}) g(x),
$$

for all $f, g \in \mathcal{H}$ and the proof of Proposition 3.9 is complete.

With Proposition 3.9 the proof of Theorem 2.2 is now complete.

\section{Commutator Estimates and Spectral Properties of $L_{\mathcal{H}_{0}}$}

In this section, we prove Proposition 3.4 and Proposition 3.5. We generalize the commutator method of Hörmander to study the spectral properties of the operator $L_{\mathcal{H}_{0}}$ which is, by Lemma 3.1, the closure of the differential operator $L$ with domain $\mathcal{C}_{0}^{\infty}(X)$ which we defined in Eq.(3.11). We recall the definition:

$$
\begin{aligned}
L & =\sum_{m=1}^{M} \frac{\lambda_{\mathrm{L}, m}^{2} \gamma_{\mathrm{L}, m}}{\beta_{\mathrm{L}}}\left(\nabla_{r_{\mathrm{L}, m}}-\beta_{\mathrm{L}} W_{\mathrm{L}, m}\right) \cdot \nabla_{r_{\mathrm{L}, m}} \\
& +\sum_{m=1}^{M} \frac{\lambda_{\mathrm{R}, m}^{2} \gamma_{\mathrm{R}, m}}{\beta_{\mathrm{R}}}\left(\nabla_{r_{\mathrm{R}, m}}-\beta_{\mathrm{R}} W_{\mathrm{R}, m}\right) \cdot \nabla_{r_{\mathrm{R}, m}} \\
& +\sum_{m=1}^{M} r_{\mathrm{L}, m} \cdot \nabla_{p_{1}}+L_{\mathrm{S}}+\sum_{m=1}^{M} r_{\mathrm{R}, m} \cdot \nabla_{p_{n}}
\end{aligned}
$$

with the abbreviations

$$
W_{\mathrm{L}, m}=\lambda_{\mathrm{L}, m}^{-2} r_{\mathrm{L}, m}-q_{1}, \quad W_{\mathrm{R}, m}=\lambda_{\mathrm{R}, m}^{-2} r_{\mathrm{R}, m}-q_{n},
$$

and where $L_{\mathrm{S}}$ is the Liouville operator associated with the Hamiltonian $H_{\mathrm{S}}(q, p)$ :

$$
L_{\mathrm{S}}=\sum_{j=1}^{n} p_{j} \cdot \nabla_{q_{j}}-\left(\nabla_{q_{j}} V\right) \cdot \nabla_{p_{j}} .
$$


For the following estimates it will be convenient to work in the flat Hilbert space $\mathrm{L}^{2}(X, \mathrm{dx})$. The differential operator $L$ is unitarily equivalent to the operator $\mathcal{K}$ on $\mathrm{L}^{2}(X, \mathrm{dx})$ with domain $\mathcal{C}_{0}^{\infty}(X)$ given by

$$
\begin{aligned}
\mathcal{K} & =e^{-\beta_{0} G / 2} L e^{\beta_{0} G / 2} \\
& =\alpha-\sum_{m=1}^{M} \frac{\lambda_{\mathrm{L}, m}^{2} \gamma_{\mathrm{L}, m}}{\beta_{\mathrm{L}}} R_{\mathrm{L}, m}^{*} R_{\mathrm{L}, m}-\sum_{m=1}^{M} \frac{\lambda_{\mathrm{R}, m}^{2} \gamma_{\mathrm{R}, m}}{\beta_{\mathrm{R}}} R_{\mathrm{R}, m}^{*} R_{\mathrm{R}, m}+\mathcal{K}_{\mathrm{as}},
\end{aligned}
$$

where $\alpha$ is given by (3.10) and

$$
\begin{aligned}
R_{\mathrm{L}, m} & =\nabla_{r_{\mathrm{L}, m}}+\sqrt{\left(\beta_{\mathrm{L}}-\beta_{0} / 2\right) \beta_{0} / 2} W_{\mathrm{L}, m}, \\
R_{\mathrm{R}, m} & =\nabla_{r_{\mathrm{R}, m}}+\sqrt{\left(\beta_{\mathrm{R}}-\beta_{0} / 2\right) \beta_{0} / 2} W_{\mathrm{R}, m}, \\
\mathcal{K}_{\mathrm{as}} & =\sum_{m=1}^{M} r_{\mathrm{L}, m} \cdot \nabla_{p_{1}}+L_{\mathrm{S}}+\sum_{m=1}^{M} r_{\mathrm{R}, m} \cdot \nabla_{p_{n}} \\
& -\frac{\beta_{\mathrm{L}}-\beta_{\mathrm{R}}}{\beta_{\mathrm{L}}+\beta_{\mathrm{R}}} \sum_{m=1}^{M} \frac{\lambda_{\mathrm{L}, m}^{2} \gamma_{\mathrm{L}, m}}{2}\left(\nabla_{r_{\mathrm{L}, m}} \cdot W_{\mathrm{L}, m}+W_{\mathrm{L}, m} \cdot \nabla_{r_{\mathrm{L}, m}}\right) \\
& +\frac{\beta_{\mathrm{L}}-\beta_{\mathrm{R}}}{\beta_{\mathrm{L}}+\beta_{\mathrm{R}}} \sum_{m=1}^{M} \frac{\lambda_{\mathrm{R}, m}^{2} \gamma_{\mathrm{R}, m}}{2}\left(\nabla_{r_{\mathrm{R}, m}} \cdot W_{\mathrm{R}, m}+W_{\mathrm{R}, m} \cdot \nabla_{r_{\mathrm{R}, m}}\right) .
\end{aligned}
$$

All subsequent estimates will be valid for any $f \in \mathcal{S}(X)$ and thus for all functions in the domain of $\mathcal{K}$.

It is convenient to introduce the following notations: We introduce new variables, and recall some earlier definitions: Let $n^{\prime}=[n / 2]$ denote the integer part of $n / 2$. We define

$$
\begin{aligned}
P_{j} & =\nabla_{p_{j}}+a_{\mathrm{L}} p_{j}, \quad j=1, \ldots, n^{\prime}, \\
P_{j} & =\nabla_{p_{j}}+a_{\mathrm{R}} p_{j}, \quad j=n^{\prime}+1, \ldots, n, \\
Q_{j} & =\nabla_{q_{j}}+a_{\mathrm{L}} W_{j}(q, r), \quad j=1, \ldots, n^{\prime}, \\
Q_{j} & =\nabla_{q_{j}}+a_{\mathrm{R}} W_{j}(q, r), \quad j=n^{\prime}+1, \ldots, n, \\
R_{\mathrm{L}, m} & =\nabla_{r_{\mathrm{L}, m}}+a_{\mathrm{L}} W_{\mathrm{L}, m}(q, r), \quad m=1, \ldots, M, \\
R_{\mathrm{R}, m} & =\nabla_{r_{\mathrm{R}, m}}+a_{\mathrm{R}} W_{\mathrm{R}, m}(q, r), \quad m=1, \ldots, M,
\end{aligned}
$$


where

$$
\begin{aligned}
a_{\mathrm{L}} & =\left(\left(\beta_{\mathrm{L}}-\beta_{0} / 2\right) \beta_{0} / 2\right)^{1 / 2}, \\
a_{\mathrm{R}} & =\left(\left(\beta_{\mathrm{R}}-\beta_{0} / 2\right) \beta_{0} / 2\right)^{1 / 2}, \\
W_{1}(q, r) & =\nabla_{q_{1}} V(q)-\sum_{m=1}^{M} r_{\mathrm{L}, m}, \\
W_{j}(q, r) & =\nabla_{q_{j}} V(q), \quad j=2, \ldots, n-1, \\
W_{n}(q, r) & =\nabla_{q_{n}} V(q)-\sum_{m=1}^{M} r_{\mathrm{R}, m}, \\
W_{\mathrm{L}, m}(q, r) & =\lambda_{\mathrm{L}, m}^{-2} r_{\mathrm{L}, m}-q_{1}, \quad m=1, \ldots, M, \\
W_{\mathrm{R}, m}(q, r) & =\lambda_{\mathrm{R}, m}^{-2} r_{\mathrm{R}, m}-q_{n}, \quad m=1, \ldots, M .
\end{aligned}
$$

We next define the operators $K_{0}, K$, and $\Lambda$ which will be used in the statement of our main bound:

$$
\begin{aligned}
\Lambda & =\left(1+\sum_{j=1}^{n} P_{j}^{*} P_{j}+\sum_{j=1}^{n} Q_{j}^{*} Q_{j}+\sum_{m=1}^{M}\left(R_{\mathrm{L}, m}^{*} R_{\mathrm{L}, m}+R_{\mathrm{R}, m}^{*} R_{\mathrm{R}, m}\right)\right)^{1 / 2}, \\
K_{0} & =\mathcal{K}_{\mathrm{as}}, \\
K & =\alpha-\mathcal{K}=-K_{0}+\sum_{m=1}^{M}\left(b_{\mathrm{L}, m} R_{\mathrm{L}, m}^{*} R_{\mathrm{L}, m}+b_{\mathrm{R}, m} R_{\mathrm{R}, m}^{*} R_{\mathrm{R}, m}\right) .
\end{aligned}
$$

Here, we use

$$
b_{\mathrm{L}, m}=\lambda_{\mathrm{L}, m}^{2} \gamma_{\mathrm{L}, m} / \beta_{\mathrm{L}}, \quad b_{\mathrm{R}, m}=\lambda_{\mathrm{R}, m}^{2} \gamma_{\mathrm{R}, m} / \beta_{\mathrm{R}} .
$$

Our main estimate is

Theorem 4.1. Under the Assumptions H1, $\mathbf{H 2}$ on $V$, there are an $\varepsilon>0$ and a $C<\infty$ such that for all $f \in \mathcal{S}(X)$ one has

$$
\left\|\Lambda^{\varepsilon} f\right\| \leq C(\|K f\|+\|f\|) .
$$

Proof. The proof will be an easy consequence of the following

Proposition 4.2. There are finite constants $C_{j}, C_{j}^{\prime}$, and $C$ such that for all $f \in \mathcal{S}(X)$ one has with $n^{\prime}=[n / 2]$,

$$
\begin{array}{rlrl}
\left\|\Lambda^{\varepsilon_{j}-1} P_{j} f\right\| & \leq C_{j}(\|K f\|+\|f\|), & j=1, \ldots, n^{\prime}, \\
\left\|\Lambda^{\varepsilon_{j}-1} P_{n+1-j} f\right\| & \leq C_{j}(\|K f\|+\|f\|), & & j=1, \ldots, n-n^{\prime}, \\
\left\|\Lambda^{\varepsilon_{j}^{\prime}-1} Q_{j} f\right\| & \leq C_{j}^{\prime}(\|K f\|+\|f\|), & & j=1, \ldots, n^{\prime}, \\
\left\|\Lambda^{\varepsilon_{j}^{\prime}-1} Q_{n+j-1} f\right\| & \leq C_{j}^{\prime}(\|K f\|+\|f\|), & & j=1, \ldots, n-n^{\prime}, \\
\left\|R_{\mathrm{L}, m} f\right\|+\left\|R_{\mathrm{R}, m} f\right\| & \leq C(\|K f\|+\|f\|), & & m=1, \ldots, M,
\end{array}
$$


where $\varepsilon_{j}=4^{1-2 j}$ and $\varepsilon_{j}^{\prime}=4^{-2 j}$.

Proof of Proposition 4.2. For the $R_{i, m}$, we have the easy estimate

$$
\left\|R_{i, m} f\right\|^{2}=\left(f, R_{i, m}^{*} R_{i, m} f\right) \leq b_{i, m}^{-1} \operatorname{Re}(f, K f) \leq b_{i, m}^{-1}\|K f\|\|f\| \leq b_{i, m}^{-1}(\|K f\|+\|f\|)^{2} .
$$

This proves Eq.(4.11) for these cases.

For the other cases, the proof will proceed by induction: It will proceed by bounds on $P_{1}$, $Q_{1}, P_{2}, \ldots, Q_{n^{\prime}}$, and a totally symmetric argument, which is left to the reader, can be used from the other end of the chain, proceeding over $P_{n}, Q_{n}, P_{n-1}$, until the bounds reach the "center" of the chain. We next prepare the inductive proof. To make the result of this calculation clearer, we define the matrices

$$
\mathcal{M}_{j, k}=\nabla_{q_{j}} \nabla_{q_{k}} V, \quad j, k=1, \ldots, n .
$$

In components, this means, for $\mu, \nu \in\{1, \ldots, d\}$,

$$
\mathcal{M}_{j, k}^{(\mu, \nu)}=\nabla_{q_{j}^{(\mu)}} \nabla_{q_{k}^{(\nu)}} V, \quad j, k=1, \ldots, n .
$$

By our choice of potential $V$ all the $\mathcal{M}_{j, k}$ vanish, except $\mathcal{M}_{j, j}$, with $j=1, \ldots, n$ and $\mathcal{M}_{j+1, j}=$ $\mathcal{M}_{j, j+1}$, with $j=1, \ldots, n-1$. Furthermore, by Condition $\mathbf{H} 1$, all the $\mathcal{M}_{j, k}^{(\mu, \nu)}$ are uniformly bounded functions of $q$. Finally, by Assumption $\mathbf{H 2}$, the matrices $\mathcal{M}_{j, j+1}$ are definite, with uniformly bounded inverse.

One verifies easily the relations:

$$
\begin{aligned}
{\left[R_{\mathrm{L}, m}, K_{0}\right] } & =P_{1}+c_{m} R_{\mathrm{L}, m}, \\
{\left[P_{1}, K_{0}\right] } & =Q_{1}, \\
{\left[Q_{1}, K_{0}\right] } & =-\mathcal{M}_{1,1} P_{1}-\mathcal{M}_{2,1} P_{2}+\sum_{m=1}^{M} c_{m}^{\prime}\left(R_{\mathrm{L}, m}+R_{\mathrm{L}, m}^{*}\right), \\
{\left[P_{j}, K_{0}\right] } & =Q_{j}, \quad j=2, \ldots, n-1, \\
{\left[Q_{j}, K_{0}\right] } & =-\mathcal{M}_{j-1, j} P_{j-1}-\mathcal{M}_{j, j} P_{j}-\mathcal{M}_{j+1, j} P_{j+1}, \quad j=2, \ldots, n-1,
\end{aligned}
$$

where

$$
\begin{aligned}
& c_{m}=\gamma_{\mathrm{L}, m}\left(\beta_{\mathrm{L}}-\beta_{0}\right) / \beta_{\mathrm{L}}, \\
& c_{m}^{\prime}=b_{\mathrm{L}, m} a_{\mathrm{L}}\left(\beta_{\mathrm{L}}-\beta_{0}\right) .
\end{aligned}
$$

Symmetrical relations hold at the other end of the chain. With these notations, we can rewrite (among several possibilities):

$$
\begin{aligned}
P_{1} & =\left[R_{\mathrm{L}, 1}, K_{0}\right]-c_{1} R_{\mathrm{L}, 1}, \\
Q_{1} & =\left[P_{1}, K_{0}\right], \\
P_{2} & =-\mathcal{M}_{2,1}^{-1}\left(\left[Q_{1}, K_{0}\right]+\mathcal{M}_{1,1} P_{1}-\sum_{m=1}^{M} c_{m}^{\prime}\left(R_{\mathrm{L}, m}+R_{\mathrm{L}, m}^{*}\right)\right), \\
Q_{j} & =\left[P_{j}, K_{0}\right], \quad j=2, \ldots, n, \\
P_{j+1} & =-\mathcal{M}_{j+1, j}^{-1}\left(\left[Q_{j}, K_{0}\right]+\mathcal{M}_{j-1, j} P_{j-1}+\mathcal{M}_{j, j} P_{j}\right), \quad j=2, \ldots, n^{\prime},
\end{aligned}
$$


with symmetrical relations at the other end of the chain. We can streamline this representation by defining $Q_{0}=R_{\mathrm{L}, 1}$, and $\mathcal{M}_{1,0}=-1$. Then we can write, for $j=1, \ldots, n^{\prime}$ :

$$
\begin{aligned}
P_{j} & =-\mathcal{M}_{j, j-1}^{-1}\left(\left[Q_{j-1}, K_{0}\right]+S_{j}\right), \\
Q_{j} & =c
\end{aligned}
$$

where the operators $S_{j}$ depend linearly on $\left\{P_{1}, \ldots, P_{j-1}\right\},\left\{Q_{1}, \ldots, Q_{j-1}\right\}$, and the $R_{\mathrm{L}, m}$. The relations Eqs.(4.15) and (4.16) will be used in the inductive proof.

Such relations are of course reminiscent of those appearing in the study of hypoelliptic operators. The novelty here will be that we obtain bounds which are valid not only in a compact domain, but in the unbounded domain of the $p$ 's and $q$ 's.

The following bounds will be used repeatedly:

Proposition 4.3. Let $Z$ denote one of the operators $Q_{j}, Q_{j}^{*}, P_{j}$, or $P_{j}^{*}$. Let $\mathcal{M}$ denote one of the $\mathcal{M}_{j, k}$. Assume that $\alpha \in(0,2)$. Then the following operators are bounded in $\mathrm{L}^{2}(X, \mathrm{dx})$ :

1) $\Lambda^{\beta}\left[\mathcal{M}, \Lambda^{-\alpha}\right] \Lambda^{\gamma}$, if $\beta+\gamma \leq \alpha+1$,

2) $\Lambda^{\beta} Z \Lambda^{\gamma}$, if $\beta+\gamma \leq-1$,

3) $\Lambda^{\beta}\left[K_{0}, Z\right] \Lambda^{\gamma}$, if $\beta+\gamma \leq-1$,

4) $\Lambda^{\beta}\left[Z, \Lambda^{-\alpha}\right] \Lambda^{\gamma}$, if $\beta+\gamma \leq \alpha+1$,

5) $\Lambda^{\beta}\left[\Lambda^{-\alpha}, K_{0}\right] \Lambda^{\gamma}$, if $\beta+\gamma \leq \alpha$.

Proof. The proof will be given in Appendix B.

Because we are working in an infinite domain, and work with non-linear couplings, we will not bound the 1.h.s of Eq.(4.7) directly, but instead the more convenient quantity ${ }^{2}$ :

$$
R_{j}(f)=\left(\Lambda^{\varepsilon_{j}-1} \mathcal{M}_{j, j-1} P_{j} f, \Lambda^{\varepsilon_{j}-1} P_{j} f\right) .
$$

We have the

Lemma 4.4. There is a constant $C$ such that for all $j \in\{1, \ldots, n\}$ and all $f \in \mathcal{S}(X)$ one has the inequality

$$
\left\|\Lambda^{\varepsilon_{j}-1} P_{j} f\right\|^{2} \leq C\left(\left|R_{j}(f)\right|+\|f\|^{2}\right) .
$$

Therefore, to prove Eq.(4.7), it suffices to prove the corresponding inequality for the $R_{j}$.

Proof of Lemma 4.4. Let $\mathcal{M}=\mathcal{M}_{j, j-1}, \varepsilon=\varepsilon_{j}$, and $P=P_{j}$. Then, by our Assumption H2, there is a constant $m>0$ for which $\mathcal{M}>m$. Therefore,

$$
\begin{aligned}
\left\|\Lambda^{\varepsilon-1} P f\right\|^{2} & =\left(\Lambda^{\varepsilon-1} P f, \Lambda^{\varepsilon-1} P f\right) \\
& \leq m^{-1}\left|\left(\mathcal{M} \Lambda^{\varepsilon-1} P f, \Lambda^{\varepsilon-1} P f\right)\right| \\
& \leq m^{-1}\left|\left(\Lambda^{\varepsilon-1} \mathcal{M} P f, \Lambda^{\varepsilon-1} P f\right)\right|+m^{-1}\left|\left(\left[\Lambda^{\varepsilon-1}, \mathcal{M}\right] P f, \Lambda^{\varepsilon-1} P f\right)\right| \\
& \leq m^{-1}\left|R_{j}(f)\right|+m^{-1}\left|\left(\left(\Lambda^{\varepsilon}\left[\Lambda^{\varepsilon-1}, \mathcal{M}\right] \Lambda\right)\left(\Lambda^{-1} P\right) f,\left(\Lambda^{-1} P\right) f\right)\right| .
\end{aligned}
$$

\footnotetext{
${ }^{2}$ For readers familiar with the method of Hörmander, we wish to point out that this device seemed necessary because we do not have good bounds on $\left[K_{0},\left[Q_{1}, K_{0}\right]\right]$.
} 
The proof of Lemma 4.4 is completed by using the bounds 1) and 2) of Proposition 4.3.

The inductive step. We begin by the induction step for the $P_{j}$. We assume now that the bounds (4.7) and (4.9) have been shown for all $j \leq k$. We want to show (4.7) for $j=k+1$. Using Eq.(4.15) and Lemma 4.4, we start by writing

$$
\begin{aligned}
R_{k+1}(f) \equiv & \left(\Lambda^{\varepsilon_{k+1}-1} \mathcal{M}_{k+1, k} P_{k+1} f, \Lambda^{\varepsilon_{k+1}-1} P_{k+1} f\right) \\
= & \left(\Lambda^{2 \varepsilon_{k+1}-1}\left[K_{0}, Q_{k}\right] f, \Lambda^{-1} P_{k+1} f\right) \\
& -\left(\Lambda^{2 \varepsilon_{k+1}-1} S_{k+1} f, \Lambda^{-1} P_{k+1} f\right) \\
\equiv & X_{1}-X_{2} .
\end{aligned}
$$

We first bound $X_{2}$. Note that $S_{k+1}$ is a sum of terms of the form $\mathcal{M} T$ where $T$ is equal to $P_{j}$ or $Q_{j}$ with $j \leq k$, and $\mathcal{M}$ is either a constant or equal to one of the $\mathcal{M}_{k, \ell}$. Therefore we obtain, using Proposition 4.3, the inductive hypothesis, and the choice $2 \varepsilon_{k+1} \leq \min _{j \leq k}\left(\varepsilon_{j}, \varepsilon_{j}^{\prime}\right)=\varepsilon_{k}^{\prime}$ :

$$
\begin{aligned}
& \left|\left(\Lambda^{2 \varepsilon_{k+1}-1} \mathcal{M} T f, \Lambda^{-1} P_{k+1} f\right)\right| \\
& \quad \leq\left|\left(\mathcal{M} \Lambda^{2 \varepsilon_{k+1}-1} T f, \Lambda^{-1} P_{k+1} f\right)\right|+\left|\left(\left(\left[\Lambda^{2 \varepsilon_{k+1}-1}, \mathcal{M}\right] \Lambda\right)\left(\Lambda^{-1} T\right) f, \Lambda^{-1} P_{k+1} f\right)\right| \\
& \quad \leq \mathcal{O}(1)(\|K f\|+\|f\|)\|f\|+\mathcal{O}(1)\|f\|^{2} \leq \mathcal{O}(1)(\|K f\|+\|f\|)^{2} .
\end{aligned}
$$

This proves the desired bound.

We now come to the "interesting" term $X_{1}$. The commutator is rewritten as

$$
\begin{aligned}
{\left[K_{0}, Q_{k}\right] } & =-Q_{k} K-K^{*} Q_{k}+\frac{1}{2}\left(Q_{k}\left(K+K^{*}\right)+\left(K+K^{*}\right) Q_{k}\right) \\
& \equiv X_{3}+X_{4}+X_{5}
\end{aligned}
$$

We discuss the 3 corresponding bounds:

Term $X_{3}$. In this case, we are led to bound, with $\varepsilon=\varepsilon_{k+1}$,

$$
\begin{aligned}
T_{3} & \equiv\left|\left(Q_{k} K f, \Lambda^{2 \varepsilon-2} P_{k+1} f\right)\right|=\left|\left(K f, Q_{k}^{*} \Lambda^{2 \varepsilon-2} P_{k+1} f\right)\right| \\
& =\left|\left(K f,\left(Q_{k}^{*} \Lambda^{2 \varepsilon-1}\right)\left(\Lambda^{-1} P_{k+1}\right) f\right)\right| \\
& \leq\left|\left(K f,\left(\Lambda^{-1} P_{k+1}\right)\left(Q_{k}^{*} \Lambda^{2 \varepsilon-1}\right) f\right)\right|+\left|\left(K f,\left[Q_{k}^{*} \Lambda^{2 \varepsilon-1}, \Lambda^{-1} P_{k+1}\right] f\right)\right| \\
& \equiv X_{3,1}+X_{3,2} .
\end{aligned}
$$

We start by bounding $X_{3,1}$. Since $\Lambda^{-1} P_{k+1}$ is bounded by Proposition 4.3, it suffices to show that

$$
\left\|Q_{k}^{*} \Lambda^{2 \varepsilon-1} f\right\| \leq C(\|K f\|+\|f\|) .
$$


To see this we first write, using $Q=Q_{k}$,

$$
\begin{aligned}
& \left\|Q^{*} \Lambda^{2 \varepsilon-1} f\right\|^{2}=\left(f, \Lambda^{2 \varepsilon-1} Q Q^{*} \Lambda^{2 \varepsilon-1} f\right) \\
& =\left\|\Lambda^{2 \varepsilon-1} Q f\right\|^{2}+\left(f,\left[\Lambda^{2 \varepsilon-1} Q, Q^{*} \Lambda^{2 \varepsilon-1}\right] f\right) .
\end{aligned}
$$

The first term is bounded by the inductive hypothesis by $\mathcal{O}(1)(\|K f\|+\|f\|)^{2}$ and the choice of $\varepsilon_{k+1}$, while the second can be bounded by $\mathcal{O}(1)\|f\|^{2}$ by expanding the commutator (and using Proposition 4.3):

$$
\begin{gathered}
{\left[\Lambda^{2 \varepsilon-1} Q, Q^{*} \Lambda^{2 \varepsilon-1}\right]=\left(\Lambda^{2 \varepsilon-1} Q^{*} \Lambda^{-2 \varepsilon}\right) \Lambda^{2 \varepsilon}\left[Q, \Lambda^{2 \varepsilon-1}\right]} \\
+\Lambda^{2 \varepsilon-1}\left[Q, Q^{*}\right] \Lambda^{2 \varepsilon-1}+\left(\left[\Lambda^{2 \varepsilon-1}, Q^{*}\right] \Lambda^{2 \varepsilon}\right) \Lambda^{-1} Q
\end{gathered}
$$

This proves Eq.(4.18).

To bound $X_{3,2}$, we use $\left[P_{k+1}^{*}, Q_{k}\right]=0$ and we write

$$
\left[Q_{k}^{*} \Lambda^{2 \varepsilon-1}, \Lambda^{-1} P_{k+1}\right]=\left(Q_{k}^{*} \Lambda^{-1}\right)\left[\Lambda^{2 \varepsilon-1}, P_{k+1}\right]+\left(\left[Q_{k}^{*}, \Lambda^{-1}\right] \Lambda^{2 \varepsilon}\right)\left(\Lambda^{-2 \varepsilon} P_{k+1} \Lambda^{2 \varepsilon-1}\right) .
$$

Since each factor above is bounded by Proposition 4.3, the desired bound follows:

$$
T_{3} \leq \mathcal{O}(1)(\|K f\|+\|f\|)^{2} .
$$

Term $X_{4}$. Here, we want to bound $T_{4} \equiv\left|\left(K^{*} Q_{k} f, \Lambda^{2 \varepsilon-2} P_{k+1} f\right)\right|$. We get

$$
\begin{gathered}
T_{4}=\left|\left(K^{*} Q_{k} f, \Lambda^{2 \varepsilon-2} P_{k+1} f\right)\right|=\left|\left(Q_{k} f, K \Lambda^{2 \varepsilon-2} P_{k+1} f\right)\right| \\
\leq\left|\left(\Lambda^{2 \varepsilon-1} Q_{k} f, \Lambda^{-1} P_{k+1} K f\right)\right|+\left|\left(Q_{k} f,\left[K, \Lambda^{2 \varepsilon-2} P_{k+1}\right] f\right)\right| \\
\equiv X_{4,1}+X_{4,2} .
\end{gathered}
$$

Using the inductive hypothesis, and the bound $\left\|\Lambda^{-1} P_{k+1}\right\| \leq \mathcal{O}(1)$, the term $X_{4,1}$ is bounded by

$$
\left\|\Lambda^{2 \varepsilon-1} Q_{k} f\right\|\left\|\Lambda^{-1} P_{k+1} K f\right\| \leq \mathcal{O}(1)(\|K f\|+\|f\|)^{2} .
$$

We write the commutator of $X_{4,2}$ as

$$
\left[K, \Lambda^{2 \varepsilon-2} P_{k+1}\right]=\Lambda^{2 \varepsilon-1}\left(\Lambda^{-1}\left[K, P_{k+1}\right]+\Lambda^{-1}\left[K_{0}, \Lambda^{2-2 \varepsilon}\right] \Lambda^{2 \varepsilon-1}\left(\Lambda^{-1} P_{k+1}\right)\right),
$$

since $K-K_{0}$ commutes with $\Lambda$. Using Proposition 4.3 and the inductive hypothesis this leads to the following bound for $X_{4,2}$ :

$$
\begin{aligned}
X_{4,2} \leq & \left|\left(\Lambda^{2 \varepsilon-1} Q_{k} f, \Lambda^{-1}\left[K, P_{k+1}\right] f\right)\right| \\
& +\left|\left(\Lambda^{2 \varepsilon-1} Q_{k} f,\left(\Lambda^{-1}\left[K_{0}, \Lambda^{2-2 \varepsilon}\right] \Lambda^{2 \varepsilon-1}\right)\left(\Lambda^{-1} P_{k+1}\right) f\right)\right| \\
\leq & \mathcal{O}(1)(\|K f\|+\|f\|)(\|f\|) .
\end{aligned}
$$


This completes the bounds involving $X_{4}$.

Term $X_{5}$. Here, we bound

$$
T_{5} \equiv \frac{1}{2}\left(\left(Q_{k}\left(K+K^{*}\right)+\left(K+K^{*}\right) Q_{k}\right) f, \Lambda^{2 \varepsilon-2} P_{k+1} f\right) .
$$

Assume first $k>1$ (and in any case we have $k<n$ ). Looking at the definition of $K$, we see that in this case $Q_{k}$ commutes with $\frac{1}{2}\left(K+K^{*}\right)=\operatorname{Re} K$, and we can rewrite $T_{5}$ as

$$
T_{5}^{\prime}=2\left((\operatorname{Re} K) f, Q_{k}^{*} \Lambda^{2 \varepsilon-2} P_{k+1} f\right) .
$$

Using the Schwarz inequality and the positivity of $\operatorname{Re} K$, we get a bound

$$
\begin{aligned}
\left|T_{5}^{\prime}\right| & \leq((\operatorname{Re} K) f, f)^{1 / 2}\left((\operatorname{Re} K) Q_{k}^{*} \Lambda^{2 \varepsilon-2} P_{k+1} f, Q_{k}^{*} \Lambda^{2 \varepsilon-2} P_{k+1} f\right)^{1 / 2} \\
& =(\operatorname{Re}(K f, f))^{1 / 2}\left(\operatorname{Re}\left(K Q_{k}^{*} \Lambda^{2 \varepsilon-2} P_{k+1} f, Q_{k}^{*} \Lambda^{2 \varepsilon-2} P_{k+1} f\right)\right)^{1 / 2} \\
& =(\operatorname{Re}(K f, f))^{1 / 2}\left(\operatorname{Re}\left(\Lambda^{-2 \varepsilon} K Q_{k}^{*} \Lambda^{2 \varepsilon-2} P_{k+1} f, \Lambda^{2 \varepsilon} Q_{k}^{*} \Lambda^{2 \varepsilon-2} P_{k+1} f\right)\right)^{1 / 2} \\
& \equiv(\operatorname{Re}(K f, f))^{1 / 2}\left(\operatorname{Re}\left(f_{1}, f_{2}\right)\right)^{1 / 2} .
\end{aligned}
$$

The first factor is clearly bounded by $(\|K f\|+\|f\|)^{1 / 2}$. To bound $f_{1}$, we expand again:

$$
\begin{aligned}
f_{1} & =\Lambda^{-2 \varepsilon} K Q^{*} \Lambda^{2 \varepsilon-2} P f=\left(\Lambda^{-2 \varepsilon} Q^{*} \Lambda^{2 \varepsilon-1}\right)\left(\Lambda^{-1} P\right) K f \\
& +\Lambda^{-2 \varepsilon}\left[K, Q^{*}\right] \Lambda^{2 \varepsilon-2} P f+\Lambda^{-2 \varepsilon} Q^{*}\left[K, \Lambda^{2 \varepsilon-2}\right] P f \\
& +\Lambda^{-2 \varepsilon} Q^{*} \Lambda^{2 \varepsilon-2}[K, P] f .
\end{aligned}
$$

The norm of the first term is bounded by $\mathcal{O}(1)(\|K f\|+\|f\|)$. Using Proposition 4.3, the other terms are bounded by $\mathcal{O}(1)\|f\|$. To bound $f_{2}$ we write

$$
\begin{aligned}
f_{2} & =\Lambda^{2 \varepsilon} Q^{*} \Lambda^{2 \varepsilon-2} P f=\Lambda^{-1} P Q^{*} \Lambda^{4 \varepsilon-1} f \\
& +\Lambda^{2 \varepsilon} Q^{*} \Lambda^{-2 \varepsilon-1}\left[\Lambda^{4 \varepsilon-1}, P\right] f \\
& +\Lambda^{2 \varepsilon}\left[Q^{*}, \Lambda^{-2 \varepsilon-1}\right] P \Lambda^{4 \varepsilon-1} f .
\end{aligned}
$$

We control the first term using the inductive hypothesis (it is here that we use the factor $4 \varepsilon_{k+1} \leq \varepsilon_{k}^{\prime}$ ) and the two others by Proposition 4.3. Combining these bounds, we finally get the bound $T_{5} \leq \mathcal{O}(1)(\|K f\|+\|f\|)^{2}$, and hence the inequality (4.7) is shown for all $j$.

It remains to discuss the cases $k=0,1$ for the term $X_{5}$. The commutators of $\operatorname{Re} K$ with $Q_{0} \equiv R_{\mathrm{L}, 1}$ or with $Q_{1}$ do not vanish and hence there are additional terms in $T_{5}^{\prime}$. They are of the form

$$
\begin{gathered}
\sum_{m=1}^{M} b_{\mathrm{L}, m}\left(\left[R_{\mathrm{L}, m}^{*} R_{\mathrm{L}, m}, R_{\mathrm{L}, 1}\right] f, \Lambda^{2 \varepsilon-2} P_{k+1} f\right), \\
\sum_{m=1}^{M} b_{\mathrm{L}, m}\left(\left[R_{\mathrm{L}, m}^{*} R_{\mathrm{L}, m}, Q_{1}\right] f, \Lambda^{2 \varepsilon-2} P_{k+1} f\right) .
\end{gathered}
$$


Since $\left[R_{\mathrm{L}, m}^{*} R_{\mathrm{L}, m}, R_{\mathrm{L}, 1}\right]=$ const. $R_{\mathrm{L}, 1} \delta_{m, 1}$ and $\left[R_{\mathrm{L}, m}^{*} R_{\mathrm{L}, m}, Q_{1}\right]=$ const. $R_{\mathrm{L}, m}$, this is obviously bounded by $\mathcal{O}(1)(\|K f\|+\|f\|)\|f\|$.

We have discussed now all the cases for the inductive bound on the $P_{j}$. The discussion of this step for the $Q_{j}$ is the same, except that some simplifications appear because of the simpler relations $Q_{j}=\left[P_{j}, K_{0}\right]$. The proof of Proposition 4.2 is complete.

Proof of Theorem 4.1. Let $\varepsilon \leq \varepsilon_{n+1}^{\prime}$. We rewrite

$$
\Lambda^{2 \varepsilon}=\Lambda^{2 \varepsilon-2}\left(1+\sum_{j=0}^{n+1} Q_{j}^{*} Q_{j}+\sum_{j=1}^{n} P_{j}^{*} P_{j}\right) .
$$

Note now that for $Q=Q_{j}$,

$$
\Lambda^{2 \varepsilon-2} Q^{*} Q=Q^{*} \Lambda^{2 \varepsilon-2} Q+\left[\Lambda^{2 \varepsilon-1}, Q^{*}\right] Q .
$$

Using Proposition 4.2 and Proposition 4.3, we get a bound

$$
\left(f, \Lambda^{2 \varepsilon-2} Q^{*} Q f\right) \leq \mathcal{O}(1)(\|K f\|+\|f\|)^{2}+\mathcal{O}(1)\|f\|^{2} .
$$

Of course, the $P$ satisfy analogous relations. Since $\left\|\Lambda^{2 \varepsilon-2} f\right\| \leq \mathcal{O}(1)\|f\|$, the assertion (4.6) follows by summing the terms in Eq.(4.20). The proof of Theorem 4.1 is complete.

Using Theorem 4.1 we can now prove Proposition 3.4. We have

Proposition 4.5. If the potential $V$ satisfies Conditions $\mathbf{H 1}, \mathbf{H} 2$ and if $\beta_{0}<2 \min \left(\beta_{\mathrm{L}}, \beta_{\mathbf{R}}\right)$ there is a $\lambda^{*}>0$ such that if the couplings satisfy $\left|\lambda_{\mathrm{L}, m}\right|,\left|\lambda_{\mathrm{R}, m}\right| \in\left(0, \lambda^{*}\right)$ then both $L_{\mathcal{H}_{0}}$ and $L_{\mathcal{H}_{0}}^{*}$ have compact resolvent.

Proof. We show that the operator $\mathcal{K}$ on $\mathrm{L}^{2}(X, \mathrm{dx})$ has compact resolvent. From Theorem 4.1 we get the bound

$$
\left\|\Lambda^{\varepsilon} f\right\| \leq C(\|(\mathcal{K}-\alpha-1) f\|+\|f\|),
$$

for all $f \in \mathcal{S}(X)$. Since, by Lemma 3.1, $\mathcal{C}_{0}^{\infty}(X)$ is a core of $\mathcal{K}$, we see, by taking limits, that the estimate (4.21) holds for all $f$ in $D(\mathcal{K})$.

We note that $\Lambda^{2}$ has compact resolvent. Indeed, recall the definition Eq.(3.7) of the effective Hamiltonian $G$. It is easily checked that, first of all, $G$ grows quadratically in every direction of $\mathbf{R}^{2 d(n+M)}$, for sufficiently small $\left|\lambda_{i, m}\right|$. Second, it is also easily verified that

$$
\Lambda^{2}=1-\sum_{j=1}^{n}\left(\Delta_{p_{j}}+\Delta_{q_{j}}\right)+\sum_{m=1}^{M}\left(\Delta_{r_{\mathrm{L}, m}}+\Delta_{r_{\mathrm{R}, m}}\right)+\mathbf{W}(p, q, r),
$$

and, by construction

$$
\begin{aligned}
\mathbf{W}(p, q, r) & \approx \sum_{j=1}^{n^{\prime}} a_{\mathrm{L}}^{2}\left(\left(\nabla_{p_{j}} G\right)^{2}+\left(\nabla_{q_{j}} G\right)^{2}\right)+\sum_{j=n^{\prime}+1}^{n} a_{\mathrm{R}}^{2}\left(\left(\nabla_{p_{j}} G\right)^{2}+\left(\nabla_{q_{j}} G\right)^{2}\right) \\
& +\sum_{m=1}^{M}\left(a_{\mathrm{L}}^{2}\left(\nabla_{r_{\mathrm{L}, m}} G\right)^{2}+a_{\mathrm{R}}^{2}\left(\nabla_{r_{\mathrm{R}, m}} G\right)^{2}\right)
\end{aligned}
$$


up to bounded terms. Thus $\mathbf{W}(p, q, r)$ diverges in all directions of $\mathbf{R}^{2 d(n+M)}$. Using the Rellich criterion (see [RS], Thm XII.67) we conclude that $\Lambda^{\varepsilon}$ has compact resolvent for every $\varepsilon>0$.

Therefore, Eq.(4.21) implies, using again the Rellich criterion, that $(\mathcal{K}-\alpha-1)^{*}(\mathcal{K}-$ $\alpha-1)$ has compact resolvent. We claim this implies that $\mathcal{K}$ itself has compact resolvent. Indeed, since $\mathcal{K}-\alpha-1$ is strictly $m$-accretive, its inverse exists, and therefore the operator $\left((\mathcal{K}-\alpha-1)^{*}(\mathcal{K}-\alpha-1)\right)^{-1}=\left((\mathcal{K}-\alpha-1)^{*}\right)^{-1}(\mathcal{K}-\alpha-1)^{-1}$ exists and is compact. This implies that $(\mathcal{K}-\alpha-1)^{-1}$ is compact and hence $\mathcal{K}$ has compact resolvent as asserted.

Finally, we prove Proposition 3.5. We have the following

Proposition 4.6. Let $g$ denote an eigenvector of $L_{\mathcal{H}_{0}}$ or $L_{\mathcal{H}_{0}}^{*}$. If the assumptions of Proposition 3.4 are satisfied then $g \exp \left(\beta_{0} G / 2\right)$ is in the Schwartz space $\mathcal{S}(X)$.

Proof. We prove the corresponding statement for the operator $\mathcal{K}$ on $\mathrm{L}^{2}(X, \mathrm{dx})$. We consider the set of $\mathcal{C}^{\infty}$ vectors of $e^{\mathcal{K} t}$, i.e the set

$$
\mathcal{C}^{\infty}(\mathcal{K}) \equiv\left\{f \in \mathrm{L}^{2}(X, \mathrm{dx}) ; e^{\mathcal{K} t} \in \mathcal{C}^{\infty}\left(\mathbf{R}^{+}, \mathrm{L}^{2}(X, \mathrm{dx})\right)\right\} .
$$

The set $\mathcal{C}^{\infty}(\mathcal{K})$ obviously contains all eigenvectors of $\mathcal{K}$. Therefore Proposition 4.6 is a direct consequence of the following Proposition.

Proposition 4.7. $\mathcal{C}^{\infty}(\mathcal{K})=\mathcal{S}(X)$.

Proof. By Theorem 1.43 in [Da] we have the following characterization of $\mathcal{C}^{\infty}(\mathcal{K})$ :

$$
\mathcal{C}^{\infty}(\mathcal{K})=\cap_{n \geq 0} D\left(\mathcal{K}^{n}\right)
$$

where $D\left(\mathcal{K}^{n}\right)=\left\{f \in D\left(\mathcal{K}^{n-1}\right), \mathcal{K}^{n-1} f \in D(\mathcal{K})\right\}$.

Since $\mathcal{S}(X) \subset D(\mathcal{K})$ and $\mathcal{K} \mathcal{S}(X) \subset \mathcal{S}(X)$, we have the easy inclusion

$$
\mathcal{S}(X) \subset \cap_{n} D\left(\mathcal{K}^{n}\right)=\mathcal{C}^{\infty}(\mathcal{K})
$$

To show the inclusion in the other direction we will need the following Theorem which we will prove in Appendix C. This is a (slight) generalization of the core Theorem, [Da], Thm 1.9.

Theorem 4.8. Let $\mathcal{B}$ be a Banach space. Let $A: D(A) \rightarrow \mathcal{B}$ be $m$-accretive. For all $n=1,2, \ldots$, if $\mathcal{D}$ is a subset of $D\left(A^{n}\right)$ and is dense in $\mathcal{B}$ and furthermore $\mathcal{D}$ is invariant under the semi-group $e^{A t}$, then $\mathcal{D}$ is a core for $A^{n}$.

Given this result we first show that $\mathcal{S}(X)$ is invariant under $e^{\mathcal{K} t}$. For $s \geq 0$ we consider the scale of spaces $\mathcal{N}_{s}$ given by $\mathcal{N}_{s}=D\left(\Lambda^{s}\right)$, with the norm $\|f\|_{(s)}=\left\|\Lambda^{s} f\right\|$. For $s \leq 0$ we let $\mathcal{N}_{s}$ be the dual of $\mathcal{N}_{-s}$. From the definition of $\Lambda^{2}$, it is easy to see that $\left\{\|\cdot\|_{(s)} ; s=0,1, \cdots\right\}$ is a system of semi-norms for the topology of $\mathcal{S}(X)$ and hence $\mathcal{S}(X)=\cap_{s} \mathcal{N}_{s}$.

To show that $\mathcal{S}(X)$ is left invariant by the semi-group $e^{\mathcal{K} t}$ generated by $\mathcal{K}$, it is enough to show that

$$
e^{\mathcal{K} t} \mathcal{N}_{s} \subset \mathcal{N}_{s} \quad \text { for all } s \geq 0 .
$$


For $f, g$ in $\mathcal{S}(X)$ we have the identity

$$
\begin{aligned}
\left(\Lambda^{-s} e^{\mathcal{K}^{*}}{ }^{s} f, g\right) & =\left(f, \Lambda^{s} e^{\mathcal{K} t} \Lambda^{-s} g\right) \\
& =(f, g)+\int_{0}^{t} \mathrm{~d} \tau\left(f, \Lambda^{s} \mathcal{K} e^{\mathcal{K} \tau} \Lambda^{-s} g\right) \\
& =(f, g)+\int_{0}^{t} \mathrm{~d} \tau\left(f,(\mathcal{K}+B) \Lambda^{s} e^{\mathcal{K} \tau} \Lambda^{-s} g\right) \\
& =(f, g)+\int_{0}^{t} \mathrm{~d} \tau\left(\Lambda^{-s} e^{\mathcal{K}^{*} \tau} \Lambda^{s}\left(\mathcal{K}^{*}+B^{*}\right) f, g\right),
\end{aligned}
$$

where

$$
B=\left[\Lambda^{s}, \mathcal{K}\right] \Lambda^{-s},
$$

is a bounded operator by Proposition 4.3. From (4.22) we see that

$$
\frac{\mathrm{d}}{\mathrm{d} t} \Lambda^{-s} e^{\mathcal{K}^{*} t} \Lambda^{s} f=\Lambda^{-s} e^{\mathcal{K}^{*} t} \Lambda^{s}\left(\mathcal{K}^{*}+B^{*}\right) f .
$$

Now $\mathcal{K}^{*}$ is the generator of a strongly continuous quasi-bounded semi-group, $B^{*}$ is bounded and so, [Ka], Chap.9, Thm 2.7, $\mathcal{K}^{*}+B^{*}$ with domain $D\left(\mathcal{K}^{*}\right)$ is the generator a strongly continuous quasi-bounded semi-group $e^{\left(\mathcal{K}^{*}+B^{*}\right) t}$ with $\left\|e^{\left(\mathcal{K}^{*}+B^{*}\right) t}\right\| \leq e^{\left(\alpha+\left\|B^{*}\right\|\right) t}$. From (4.23) we see that

$$
e^{\left(\mathcal{K}^{*}+B^{*}\right) t}=\Lambda^{-s} e^{\mathcal{K}^{*} t} \Lambda^{s} .
$$

Thus we obtain

$$
\left\|\Lambda^{-s} e^{\mathcal{K}^{*} t} \Lambda^{s}\right\| \leq e^{\left(\alpha+\left\|B^{*}\right\|\right) t},
$$

and so $e^{\mathcal{K}^{*} t}: \mathcal{N}_{-s} \rightarrow \mathcal{N}_{-s}, s>0$, is bounded. By duality $e^{\mathcal{K} t}: \mathcal{N}_{s} \rightarrow \mathcal{N}_{s}, s>0$, is also bounded. This implies that

$$
e^{\mathcal{K} t} \mathcal{N}_{s} \subset \mathcal{N}_{s}, \quad s>0,
$$

and therefore $\mathcal{S}(X)$ is invariant under $e^{\mathcal{K} t}$.

We now use Theorem 4.1. Let $f \in \mathcal{S}(X)$, then replacing $f$ by $\Lambda^{m} f$ in Eq.(4.6), we obtain

$$
\begin{aligned}
\|f\|_{(m+\varepsilon)} & \leq \mathcal{O}(1)\left(\left\|\mathcal{K} \Lambda^{m} f\right\|+\|f\|_{(m)}\right) \\
& \leq \mathcal{O}(1)\left(\|\mathcal{K} f\|_{(m)}+\left\|\left[K, \Lambda^{m}\right] f\right\|+\|f\|_{(m)}\right) .
\end{aligned}
$$

Since

$$
\left\|\left[\mathcal{K}, \Lambda^{m}\right] f\right\|=\left\|\Lambda^{m}\left[\mathcal{K}, \Lambda^{-m}\right] \Lambda^{m} f\right\|,
$$

and since $\Lambda^{m}\left[\mathcal{K}, \Lambda^{-m}\right]$ is bounded by Proposition 4.3 we obtain the bound

$$
\|f\|_{(m+\varepsilon)} \leq \mathcal{O}(1)\left(\|\mathcal{K} f\|_{(m)}+\|f\|_{(m)}\right) .
$$


Using (4.24) it is easy to see, by induction, that, for $n=1,2, \cdots$ we have

$$
\|f\|_{(n \varepsilon)} \leq \mathcal{O}(1) \sum_{j=0}^{n}\left(\begin{array}{l}
n \\
j
\end{array}\right)\left\|\mathcal{K}^{j} f\right\| .
$$

Since $\mathcal{S}(X)$ is a core for $\mathcal{K}^{n}$ by Theorem 4.8 , we see, by taking limits, that

$$
D\left(\mathcal{K}^{n}\right) \subset \mathcal{N}_{n \varepsilon} .
$$

Therefore

$$
\mathcal{C}^{\infty}(\mathcal{K})=\cap_{n} D\left(\mathcal{K}^{n}\right) \subset \cap_{n} \mathcal{N}_{n \varepsilon}=\mathcal{S}(X) .
$$

And this concludes the proof of Proposition 4.7.

\section{Appendix A: Proof of Lemma 3.1}

If $x(t)=\xi(t, w ; x)$ denotes the solution of (3.1), it has the cocycle property

$$
\xi\left(t, \tau^{s} w ; \xi(s, w ; x)\right)=\xi(t+s, w ; x),
$$

which holds for all $t, s \in \mathbf{R}, x \in X$ and $w \in \mathcal{W}$. Here we have introduced the shift $\left(\tau^{t} w\right)(s)=w(t+s)$ on $\mathcal{W}$. In particular the map $x \mapsto \xi(t, w ; x)$ is a bijection with inverse $x \mapsto \xi\left(-t, \tau^{t} w ; x\right)$. A standard argument shows that these maps are actually diffeomorphisms (see e.g., [IW], Ch. V.2). The Jacobian of $\xi(t, w ; \cdot)$ is given by

$$
J(t, w ; \cdot)=\left|\operatorname{det} D_{x} \xi(t, w ; \cdot)\right|=e^{\int_{0}^{t} \mathrm{~d} s \operatorname{div} b \circ \xi(s, w ; \cdot)},
$$

and according to (3.2) the Jacobian satisfies

$$
e^{-B|t|} \leq J(t, w ; x) \leq e^{B|t|} .
$$

Remark. In our case we have in fact

$$
\operatorname{div} b=-d \sum_{i, m} \gamma_{i, m} \equiv-\Gamma<0,
$$

so that

$$
J(t, w ; \cdot)=e^{-\Gamma t} .
$$

The Lemma 3.1 is an immediate consequence of the following lemmata. 
Lemma A.1. $T^{t}$ extends to a strongly continuous, quasi-bounded semi-group of positivity preserving operators on $\mathrm{L}^{2}(X, \mathrm{dx})$. Its generator is the closure of $L$.

Proof. Let $f \in \mathcal{C}_{0}^{\infty}$, then we have

$$
\begin{aligned}
\left\|T^{t} f\right\|^{2} & =\lim _{R \rightarrow \infty} \int_{|x|<R} \mathrm{dx}\left|T^{t} f(x)\right|^{2}=\lim _{R \rightarrow \infty} \int \mathrm{dx} \chi_{\{|x|<R\}}|\mathbf{E}[f \circ \xi(t, w ; x)]|^{2} \\
& \leq \lim _{R \rightarrow \infty} \int \mathrm{dx} \chi_{\{|x|<R\}} \mathbf{E}\left[|f|^{2} \circ \xi(t, w ; x)\right] \\
& \leq \lim _{R \rightarrow \infty} \mathbf{E}\left[\int \mathrm{dx} \chi_{\{|x|<R\}}|f|^{2} \circ \xi(t, w ; x)\right] \\
& \leq \lim _{R \rightarrow \infty} \mathbf{E}\left[\int \mathrm{dy} \chi_{\{|x|<R\}} \circ \xi\left(-t, \tau^{t} w ; y\right)|f|^{2}(y) J\left(-t, \tau^{t} w ; y\right)\right] \\
& \leq \lim _{R \rightarrow \infty} \mathbf{E}\left[\int \mathrm{dy} 1|f|^{2}(y) e^{B t}\right]=e^{B t}\|f\|^{2} .
\end{aligned}
$$

Thus $T^{t}$ extends to a bounded operator on $\mathrm{L}^{2}(X, \mathrm{dx})$ by continuity. A simple approximation argument shows that $T^{t}$ is weakly continuous at $t=0$, and hence, since it is obviously a semi-group, strongly continuous. Positivity is evident. Now let $f \in \mathcal{C}_{0}^{\infty}(X)$, then

$$
\left[(1 / t)\left(T^{t}-1\right) f-L f\right](x)=\int_{0}^{t} \mathrm{~d} s(1 / t)\left(T^{s}-1\right) L f(x),
$$

from which we conclude that the generator $\widetilde{L}$ of $T^{t}$ on $\mathrm{L}^{2}(X, \mathrm{dx})$ satisfies $L \subset \widetilde{L}$.

From the inequality $\operatorname{Re}(f, L f)=-\frac{1}{2}\left\|\sigma^{T} \nabla f\right\|^{2}-(f, \operatorname{div} b f) \leq B\|f\|^{2}$, and the fact that $\mathcal{C}_{0}^{\infty} \subset D\left(L^{*}\right)$ and $\operatorname{Re}\left(f, L^{*} f\right)=-\frac{1}{2}\left\|\sigma^{T} \nabla f\right\|^{2}+(f, \operatorname{div} b f) \leq B\|f\|^{2}$, one concludes that:

(i) $L$ is accretive.

(ii) The range of $(\lambda-L)$ is dense for $\operatorname{Re}(\lambda)>B$.

Hence, by the Lumer-Phillips Theorem (see for example [Da], Theorem 2.25), the closure $\bar{L}$ generates a quasi-bounded semi-group on $\mathrm{L}^{2}(X, \mathrm{dx})$. Since such generators are maximal accretive, we conclude that $\widetilde{L}=\bar{L}$. form

We shall now consider the Markov semi-group on weighted $\mathrm{L}^{2}$-spaces (such as $\mathcal{H}_{0}$ ) of the

$$
\mathcal{H}_{S}=\mathrm{L}^{2}\left(X, e^{-S} \mathrm{dx}\right),
$$

where $S \in \mathcal{C}^{\infty}(X)$, and $e^{-S} \in \mathrm{L}^{1}(X, \mathrm{dx})$ is normalized $\left(\left\|e^{-S}\right\|_{1}=1\right)$. We also assume that

$$
b_{S} \equiv D \nabla S
$$

with $D$ as in Eq.(3.3) satisfies the condition

$$
\left\|\operatorname{div} b_{S}\right\|_{\infty}<\infty
$$


The action of $T^{t}$ on $\mathcal{H}_{S}$ is obviously equivalent to that of $e^{-S / 2} T^{t} e^{S / 2}$ on $L^{2}(X, \mathrm{dx})$. For $f \in \mathcal{C}_{0}^{\infty}(X)$, Ito's formula gives

$$
\begin{aligned}
\left(e^{-S / 2} T^{t} e^{S / 2}\right) f(x) & =\mathbf{E}\left[e^{(S(x(t))-S(x(0))) / 2} f(x(t)) \mid x(0)=x\right] \\
& =\mathbf{E}\left[e^{\frac{1}{2} \int_{0}^{t} \mathrm{~d} s(L S)(x(s))+\frac{1}{2} \int_{0}^{t} \mathrm{~d} w(s)\left(\sigma^{T} \nabla S\right)(x(s))} f(x(t)) \mid x(0)=x\right] \\
& =\mathbf{E}\left[D(t) e^{\frac{1}{2} \int_{0}^{t} \mathrm{~d} s R_{S}(x(s))} f(x(t)) \mid x(0)=x\right],
\end{aligned}
$$

where

$$
\begin{aligned}
D(t) & =e^{\int_{0}^{t} \mathrm{~d} w(s) \frac{1}{2}\left(\sigma^{T} \nabla S\right)(x(s))-\frac{1}{2} \int_{0}^{t} \mathrm{~d} s\left|\frac{1}{2} \sigma^{T} \nabla S\right|^{2}(x(s))} \\
R_{S}(x) & =(L S)(x)+\frac{1}{2}(\nabla S \cdot D \nabla S)(x)=\operatorname{div}\left(b_{S}\right)+\left(b+\frac{1}{2} b_{S}\right) \cdot \nabla S .
\end{aligned}
$$

By the Girsanov formula we obtain

$$
\left(e^{-S / 2} T^{t} e^{S / 2} f\right)(x)=\mathbf{E}\left[e^{\frac{1}{2} \int_{0}^{t} \mathrm{~d} s R_{S}(y(s))} f(y(t)) \mid y(0)=x\right],
$$

where $y(t)$ is the Markovian diffusion process defined by the equation

$$
y(t)=y(0)+\int_{0}^{t} \mathrm{~d} s\left(b+b_{S}\right)(y(s))+\sigma(w(t)-w(0)) .
$$

Assuming that $R_{S}$ is bounded above:

$$
\Sigma_{S}=\sup _{x \in X} R_{S}(x)<\infty,
$$

and denoting by $T_{S}^{t}$ the semi-group on $\mathrm{L}^{2}(X, \mathrm{dx})$ associated with the process $y(t)$, by Lemma A.1 we get

$$
\left|\left(e^{-S / 2} T^{t} e^{S / 2} f\right)(x)\right| \leq e^{\frac{1}{2} \Sigma_{S} t}\left(T_{S}^{t}|f|\right)(x),
$$

from which one concludes that $e^{-S / 2} T^{t} e^{S / 2}$ extends to a strongly continuous, quasi-bounded semi-group of positivity preserving operators on $L^{2}(X, \mathrm{dx})$.

By the Feynman-Kac formula (or Cameron-Martin) we can conclude that the generator of this semi-group is given, on $\mathcal{C}_{0}^{\infty}(X)$, by

$$
L_{S}=\nabla \cdot D \nabla+\left(b+b_{S}\right) \cdot \nabla+\frac{1}{2} R_{S}=e^{-S / 2} L e^{S / 2} .
$$

Repeating the argument of Lemma A.1 we conclude that $\bar{L}_{S}$ is the generator. Since $\mathcal{C}_{0}^{\infty}(X)$ is invariant by $e^{ \pm S / 2}$ we get

Lemma A.2. Let $S \in \mathcal{C}^{\infty}(X)$ be such that

(i) $b_{S}=D \nabla S$ satisfies Condition (A.1), 
(ii) $\sup _{x \in X}\left(b+\frac{1}{2} b_{S}\right) \cdot \nabla S(x)<\infty$.

Then the semi-group $T^{t}$ extends to a strongly continuous quasi-bounded semi-group on $\mathcal{H}_{S}$. Moreover $\mathcal{C}_{0}^{\infty}(X)$ is a core for its generator.

Now Lemma 3.1 is a direct consequence of Lemma A.2. Indeed we apply Lemma A.2 to

$$
S(x)=\beta_{0} G(p, q, r)
$$

where $G$ is given by (3.7). We see that Condition (i) of Lemma A.2 is satisfied, since $G(p, q, r)$ is of the form quadratic + bounded. An explicit computation shows that the assumption

$$
\beta_{0}<2 \min \left(\beta_{\mathrm{L}}, \beta_{\mathrm{R}}\right)
$$

implies that Condition (ii) of Lemma A.2 is satisfied and that the semi-group satisfies the bound $\left\|T^{t}\right\| \leq e^{\alpha t}$ where $\alpha$ is given by Eq.(3.10).

\section{Appendix B: Proof of Proposition 4.3}

To prove the claims it is useful to introduce some machinery which replaces the pseudodifferential calculus, which seems unavailable for the class of operators we want to consider. This may be useful in its own right.

Let $\mathcal{F}$, as in the Hypotheses $\mathbf{H 1}, \mathbf{H} 2$ denote the class of functions of $q \in \mathbf{R}^{d n}$ which are bounded together with all their derivatives. Let $\mathcal{Y}$ denote the linear space of operators spanned by

$$
f(q) q^{m} \partial_{q}^{m^{\prime}} p^{n} \partial_{p}^{n^{\prime}} r^{\ell} \partial_{r}^{\ell^{\prime}}
$$

where $f \in \mathcal{F}$. (The notation is sloppy, we really mean components $\nu=1, \ldots, d$ of each of these quantities.) We shall say that the quantities in Eq.(B.1) are of degree $m+m^{\prime}+n+n^{\prime}+\ell+\ell^{\prime}$. We let $\mathcal{Y}^{s}$ denote the subspace of $\mathcal{Y}$ spanned by the expressions of degree less than or equal to $s$. Clearly, the operators $Z$ and $\mathcal{M}$ of Proposition 4.3 are in $\mathcal{Y}^{1}$ and $\mathcal{Y}^{0}$, respectively, while $K_{0}$ and $\Lambda^{2}$ are in $\mathcal{Y}^{2}$. Below, we shall use this, but also an additional property of the potential $V$. We have

Lemma B.1. One has the inclusion

$$
\left[\Lambda^{2}, \mathcal{Y}^{s}\right] \subseteq \mathcal{Y}^{s+1}
$$

Furthermore, $Y \in \mathcal{Y}^{0}$ defines a bounded operator.

Proof. By inspection.

Proposition B.2. Assume that $Y \in \mathcal{Y}^{j}$, for some $j \in\{0,1, \ldots\}$. Then

$$
\Lambda^{\beta} Y \Lambda^{\gamma}
$$


defines a bounded operator on $\mathrm{L}^{2}(X, \mathrm{dx})$, when

$$
\beta+\gamma \leq-j .
$$

Let $Z$ be an operator in $\mathcal{Y}$. Assume that $\left[\Lambda^{2}, Z\right] \in \mathcal{Y}^{j}$, for some $j \in\{0,1, \ldots\}$. Then

$$
\Lambda^{\beta}\left[\Lambda^{-\alpha}, Z\right] \Lambda^{\gamma}
$$

defines a bounded operator on $\mathrm{L}^{2}(X, \mathrm{dx})$ for all $\alpha, \beta$ and $\gamma$ satisfying

$$
\beta+\gamma \leq \alpha-j+2 .
$$

We will give bounds on various quantities involving $\mathcal{Y}^{s}$. For this, we will use throughout the following device:

Lemma B.3. Let $A_{z}$ be a bounded continuous operator-valued function of $z$ and let $F(\lambda, z)$ be a real, positive continuous bounded function. Then

$$
\left\|\int_{0}^{\infty} \mathrm{d} z A_{z} F(\Lambda, z) u\right\| \leq \sup _{y \geq 0}\left\|A_{y}\right\|\|u\| \int_{0}^{\infty} \mathrm{d} z \sup _{\lambda \geq 1} F(\lambda, z) .
$$

If furthermore $A=A_{z}$ is independent of $z$, one has the bound

$$
\left\|\int_{0}^{\infty} \mathrm{d} z A F(\Lambda, z) u\right\| \leq\|A\|\|u\| \sup _{\lambda \geq 1} \int_{0}^{\infty} \mathrm{d} z F(\lambda, z) .
$$

Proof. Note first that

$$
\left\|\int_{0}^{\infty} \mathrm{d} z A_{z} F(\Lambda, z) u\right\| \leq \int_{0}^{\infty} \mathrm{d} z\left\|A_{z}\right\|\|F(\Lambda, z) u\| .
$$

Since $\Lambda$, as an operator, satisfies $\Lambda \geq 1$ we also have from the spectral theorem:

$$
\|F(\Lambda, z)\| \leq \sup _{\lambda \geq 1} F(\lambda, z) .
$$

Thus, Eq.(B.5) follows. In a similar way:

$$
\left\|\int_{0}^{\infty} \mathrm{d} z A F(\Lambda, z) u\right\| \leq\|A\|\left\|\int_{0}^{\infty} \mathrm{d} z F(\Lambda, z)\right\|\|u\| \leq\|A\|\|u\| \sup _{\lambda \geq 1} \int_{0}^{\infty} \mathrm{d} z F(\lambda, z),
$$

which is (B.6). The proof of Lemma B.3 is complete.

We shall also make use of the following identity, valid for $\alpha \in(0,2)$, [Ka] Thm. V.3:

$$
\Lambda^{-\alpha}=\frac{\sin (\pi \alpha / 2)}{\pi} \int_{0}^{\infty} \mathrm{d} z z^{-\alpha / 2}\left(z+\Lambda^{2}\right)^{-1} .
$$


We also let $C_{-\alpha}=\sin (\pi \alpha / 2) / \pi$.

Proof of Proposition B.2. It is obvious that if we show the claim for $\beta+\gamma=-j$, then it also follows for $\beta+\gamma<-j$. By the definition of $\mathcal{Y}^{j}$, and observing that $f(q)$ is bounded, and by the explicit form of $\Lambda^{2}$, we see that the claim holds when $\gamma=0$. We next consider the case $\beta+\gamma \leq 0,-1 \leq \gamma<0$. In this case we write

$$
\Lambda^{\beta} Y \Lambda^{\gamma}=Y \Lambda^{\beta+\gamma}+\Lambda^{\beta}\left[Y, \Lambda^{\gamma}\right] .
$$

The first term is clearly bounded as in the case $\gamma=0$, by considering adjoints. The second term can be written as

$$
\begin{aligned}
\Lambda^{\beta}\left[Y, \Lambda^{\gamma}\right] & =C_{\gamma} \int_{0}^{\infty} \mathrm{d} z z^{\gamma / 2} \Lambda^{\beta}\left[Y, \frac{1}{z+\Lambda^{2}}\right] \\
& =C_{\gamma} \int_{0}^{\infty} \mathrm{d} z z^{\gamma / 2} \frac{\Lambda^{\beta}}{z+\Lambda^{2}}\left[Y, \Lambda^{2}\right] \frac{1}{z+\Lambda^{2}} .
\end{aligned}
$$

By Lemma B.1, we see that $\left[Y, \Lambda^{2}\right] \in \mathcal{Y}^{j+1}$ and thus, we get, using Eq.(B.5),

$$
\left\|\Lambda^{\beta}\left[Y, \Lambda^{\gamma}\right]\right\| \leq C_{\gamma} \sup _{y \geq 0}\left\|\frac{\Lambda^{\beta}}{y+\Lambda^{2}}\left[Y, \Lambda^{2}\right]\right\| \int_{0}^{\infty} \mathrm{d} z z^{\gamma / 2} \sup _{\lambda \geq 1} \frac{1}{z+\lambda^{2}} .
$$

The norm is bounded because $\gamma \in[-1,0)$ and thus $\beta-2+j+1 \leq \beta+\gamma+j=0$. The sup over $\lambda$ is $(1+z)^{-1}$ and the integral converges because $\gamma \in[-1,0)$.

We now proceed to the other choices of $\gamma$ by induction. We first deal with negative $\gamma$. Assume we have shown that $\Lambda^{\beta} Y \Lambda^{\gamma^{\prime}}$ is bounded for all $\gamma^{\prime} \in[-\tau, 0]$, and assume that $\gamma \in[-\tau-1,-\tau)$, and $Y \in \mathcal{Y}^{j}$. We write

$$
\Lambda^{\beta} Y \Lambda^{\gamma}=\Lambda^{\beta-1} Y \Lambda^{\gamma+1}+\Lambda^{\beta}\left[Y, \Lambda^{-1}\right] \Lambda^{\gamma+1} .
$$

The first term is bounded by the inductive hypothesis. To bound the second, we apply again the method used in Eq.(B.8). Then we get

$$
\Lambda^{\beta}\left[Y, \Lambda^{-1}\right] \Lambda^{\gamma+1}=C_{-1} \int_{0}^{\infty} \mathrm{d} z z^{-1 / 2} \frac{\Lambda^{\beta}}{z+\Lambda^{2}}\left[Y, \Lambda^{2}\right] \frac{\Lambda^{\gamma+1}}{z+\Lambda^{2}} .
$$

Since $\left[Y, \Lambda^{2}\right] \in \mathcal{Y}^{j+1}$, we see from the inductive hypothesis that

$$
\sup _{y \geq 0}\left\|\Lambda^{\beta} \frac{1}{y+\Lambda^{2}}\left[Y, \Lambda^{2}\right] \Lambda^{\gamma+1}\right\| \leq\left\|\Lambda^{\beta-2}\left[Y, \Lambda^{2}\right] \Lambda^{\gamma+1}\right\|
$$

is bounded and hence, using (B.5), we can complete the inductive step.

The case $\gamma>0$ is handled by observing that $Y \in \mathcal{Y}^{j}$ implies $Y^{*} \in \mathcal{Y}^{j}$, and bounding $\Lambda^{\beta} Y \Lambda^{\gamma}$ by bounding $\Lambda^{\gamma} Y^{*} \Lambda^{\beta}$. This completes the proof of the first part of Proposition B.2. 
To prove the second part, we first consider the case $\alpha \in(0,2)$. In this case, using Eq.(B.7), we write $\Lambda^{\beta}\left[\Lambda^{-\alpha}, Z\right] \Lambda^{\gamma}$ as

$$
C_{-\alpha} \int_{0}^{\infty} \mathrm{d} z z^{-\alpha / 2} \Lambda^{\beta}\left[\frac{1}{z+\Lambda^{2}}, Z\right] \Lambda^{\gamma}=C_{-\alpha} \int_{0}^{\infty} \mathrm{d} z z^{-\alpha / 2} \frac{\Lambda^{\beta}}{z+\Lambda^{2}}\left[\Lambda^{2}, Z\right] \frac{\Lambda^{\gamma}}{z+\Lambda^{2}} .
$$

We let $B=\left[\Lambda^{2}, Z\right]$, use another commutator and rewrite (B.9) as

$$
\begin{aligned}
& C_{-\alpha} \int_{0}^{\infty} \mathrm{d} z z^{-\alpha / 2} \Lambda^{\beta} B \frac{\Lambda^{\gamma}}{\left(z+\Lambda^{2}\right)^{2}}+C_{-\alpha} \int_{0}^{\infty} \mathrm{d} z z^{-\alpha / 2} \frac{\Lambda^{\beta}}{z+\Lambda^{2}}\left[\Lambda^{2}, B\right] \frac{\Lambda^{\gamma}}{\left(z+\Lambda^{2}\right)^{2}} \\
& \equiv C_{-\alpha}\left(X_{1}+X_{2}\right) .
\end{aligned}
$$

We first bound $X_{1}$. We get, using Eq.(B.6),

$$
\begin{aligned}
\left\|X_{1} u\right\| & =\left\|\Lambda^{\beta} B \Lambda^{-j-\beta} \int_{0}^{\infty} \mathrm{d} z z^{-\alpha / 2} \frac{\Lambda^{j+\beta+\gamma}}{\left(z+\Lambda^{2}\right)^{2}} u\right\| \\
& \leq\|u\|\left\|\Lambda^{\beta} B \Lambda^{-j-\beta}\right\| \sup _{\lambda \geq 1} \int_{0}^{\infty} \mathrm{d} z z^{-\alpha / 2} \frac{\lambda^{j+\beta+\gamma}}{\left(z+\lambda^{2}\right)^{2}} \\
& \leq\|u\|\left\|\Lambda^{\beta} B \Lambda^{-j-\beta}\right\| \sup _{\lambda \geq 1} \int_{0}^{\infty} \mathrm{d} s s^{-\alpha / 2} \lambda^{2-\alpha} \frac{\lambda^{j+\beta+\gamma}}{(s+1)^{2} \lambda^{4}} .
\end{aligned}
$$

Since, by assumption, $B \in \mathcal{Y}^{j}$, the norm is bounded, and the integral is bounded because $\beta+\gamma \leq \alpha-j+2$, by assumption.

To bound $X_{2}$ we first observe that by assumption, and by Lemma B.1, $C=\left[\Lambda^{2}, B\right]$ is in $\mathcal{Y}^{j+1}$. Therefore, using Eq.(B.5) we find the following bound for $X_{2}$ :

$$
\begin{aligned}
\left\|X_{2} u\right\| & =\left\|\int_{0}^{\infty} \mathrm{d} z z^{-\alpha / 2} \frac{\Lambda^{\beta}}{z+\Lambda^{2}} C \Lambda^{1-j-\beta} \cdot \frac{\Lambda^{j-1+\beta+\gamma}}{\left(z+\Lambda^{2}\right)^{2}} u\right\| \\
& \leq\|u\| \sup _{y \geq 0}\left\|\frac{\Lambda^{\beta}}{y+\Lambda^{2}} C \Lambda^{1-j-\beta}\right\| \int_{0}^{\infty} \mathrm{d} z z^{-\alpha / 2} \sup _{\lambda \geq 1} \frac{\lambda^{j-1+\beta+\gamma}}{\left(z+\lambda^{2}\right)^{2}} .
\end{aligned}
$$

This is clearly bounded when $\beta+\gamma \leq \alpha-j+2$ and $\alpha \in(0,2)$. This completes the second part of Proposition B.2 when $\alpha \in(0,2)$. If $\alpha=0$ the assertion is trivial. The case $\alpha=2$ is handled by considering the identity:

$$
\left[\Lambda^{-2}, Z\right]=\Lambda^{-2}\left[\Lambda^{2}, Z\right] \Lambda^{-2} .
$$

The cases when $\alpha>2$ follow inductively by using the identity:

$$
\Lambda^{\beta}\left[\Lambda^{-\alpha-2}, Z\right] \Lambda^{\gamma}=\Lambda^{\beta-2}\left[\Lambda^{-\alpha}, Z\right] \Lambda^{\gamma}+\Lambda^{\beta}\left[\Lambda^{-2}, Z\right] \Lambda^{\gamma-\alpha} .
$$


The cases when $\alpha<0$ follow by similar identities The proof of Proposition B.2 is complete.

Proof of Proposition 4.3. The Proposition 4.3 is a simple consequence of Proposition B.2. Since $Z$ is in $\mathcal{Y}^{1}$, the claim 2) is covered by the bound on (B.3). We next prove 3 ). The operator $K_{0}$ is in $\mathcal{Y}^{2}$ and $Z$ is in $\mathcal{Y}^{1}$. Power counting would suggest that $\left[K_{0}, Z\right] \in \mathcal{Y}^{2}$. However, by Condition $\mathbf{H 1}$, we know that $\nabla_{q_{j}} V$ equals a linear term plus a term in $\mathcal{Y}^{0}$, and hence explicit computation shows that $\left[K_{0}, Z\right] \in \mathcal{Y}^{1}$. Hence the assertion is covered by the bound on (B.3). Since $\mathcal{M} \in \mathcal{Y}^{0}$ we see from Lemma B.1 that $\left[\mathcal{M}, \Lambda^{2}\right] \in \mathcal{Y}^{1}$, and therefore the claim 1) follows from the bound on (B.4). Using again explicit calculation and Condition $\mathbf{H} 1$ we see that $\left[Z, \Lambda^{2}\right]$ is in $\mathcal{Y}^{1}$ (and not only in $\mathcal{Y}^{2}$ ) and $\left[K_{0}, \Lambda^{2}\right]$ is in $\mathcal{Y}^{2}$ (and not only in $\mathcal{Y}^{3}$ ), and hence the cases 4) and 5) follow by applying again the bound on (B.4). The proof of Proposition 4.3 is complete.

\section{Appendix C: A Generalized Core Theorem}

We prove here the following result from Section 4:

Theorem 4.8. Let $\mathcal{B}$ be a Banach space. Let $A: D(A) \rightarrow \mathcal{B}$ be m-accretive. For all $n=1,2, \ldots$, if $\mathcal{D}$ is a subset of $D\left(A^{n}\right)$ and is dense in $\mathcal{B}$ and furthermore $\mathcal{D}$ is invariant under the semi-group $e^{A t}$, then $\mathcal{D}$ is a core for $A^{n}$.

Proof. Let $\|f\|_{n}=\sum_{j=0}^{n}\left\|A^{j} f\right\|$. Then one has

(i) $\left(D\left(A^{n}\right),\|\| \cdot \|_{n}\right)$ is complete.

(ii) $e^{A t}$ is a strongly continuous semi-group on $\left(D\left(A^{n}\right),\|\cdot \cdot\|_{n}\right)$.

The statement of the theorem is equivalent to the following: If $\overline{\mathcal{D}}^{n}$ denotes the closure of $\mathcal{D}$ in the norm $\||\cdot|\|_{n}$, then we have

$$
\overline{\mathcal{D}}^{n}=D\left(A^{n}\right) .
$$

We show this by induction. For $n=1$, this is the core Theorem, [Da], Thm 1.9. Let us assume that (C.1) holds for $n-1$. Let $f \in D\left(A^{n}\right)$, so there is a sequence $\left\{f_{m}\right\} \in \mathcal{D}$ such that

$$
\lim _{m \rightarrow \infty}\|\| f_{m}-f \|_{n-1}=0 .
$$

With the $f_{m}$ we construct a sequence which converges to $f$ in the $\|\cdot \cdot\|_{n}$ norm. We set

$$
f_{m}^{(n, t)}=\int_{0}^{t} \mathrm{~d} s \frac{(t-s)^{n-1}}{(n-1) !} e^{A s} f_{m}
$$

By the above property (ii), $e^{A s}$ is strongly continuous in $s$ in $D\left(A^{n}\right)$ and hence $f_{m}^{(n, t)} \in \overline{\mathcal{D}}^{n}$. We set

$$
f^{(n, t)}=\int_{0}^{t} \mathrm{~d} s \frac{(t-s)^{n-1}}{(n-1) !} e^{A s} f .
$$


Since $e^{A s} g$ is $n$-times differentiable in $s$ when $g \in D\left(A^{n}\right)$, we obtain, upon integrating by parts:

$$
\begin{aligned}
\left\|f_{m}^{(n, t)}-f^{(n, t)}\right\|_{n} & =\sum_{j=0}^{n-1}\left\|\int_{0}^{t} \mathrm{~d} s \frac{(t-s)^{n-1}}{(n-1) !} e^{A s} A^{j}\left(f_{m}-f\right)\right\| \\
& +\left\|\int_{0}^{t} \mathrm{~d} s \frac{(t-s)^{n-1}}{(n-1) !} e^{A s} A^{n}\left(f_{m}-f\right)\right\| \\
& =\sum_{j=0}^{n-1}\left\|\int_{0}^{t} \mathrm{~d} s \frac{(t-s)^{n-1}}{(n-1) !} e^{A s} A^{j}\left(f_{m}-f\right)\right\| \\
& +\left\|-\frac{t^{n-1}}{(n-1) !} A^{n-1}\left(f_{m}-f\right)+\int_{0}^{t} \mathrm{~d} s \frac{(t-s)^{n-2}}{(n-2) !} e^{A s} A^{n-1}\left(f_{m}-f\right)\right\| \\
& \leq C e^{\gamma t} \frac{t^{n}}{n !}\left\|f_{m}-f\right\|_{n-1}+\left(C e^{\gamma t}+1\right) \frac{t^{(n-1)}}{(n-1) !}\left\|A^{n-1}\left(f_{m}-f\right)\right\| \\
& =o(1) \quad \text { as } m \rightarrow \infty,
\end{aligned}
$$

by the inductive hypothesis. This shows that $f^{(n, t)} \in \overline{\mathcal{D}}^{n}$.

To conclude we show that $\left\|\frac{n !}{t^{n}} f^{(n, t)}-f\right\|_{n} \rightarrow 0$ as $t \rightarrow 0$. We have

$$
\begin{aligned}
\left\|\frac{n !}{t^{n}} f^{(n, t)}-f\right\|_{n} & =\sum_{j=0}^{n-1}\left\|\frac{n !}{t^{n}} \int_{0}^{t} \mathrm{~d} s \frac{(t-s)^{n-1}}{(n-1) !}\left(e^{A s}-1\right) A^{j} f\right\| \\
& +\left\|\frac{n !}{t^{n}}\left(\int_{0}^{t} \mathrm{~d} s \frac{(t-s)^{n-1}}{(n-1) !} e^{A s} A^{n} f-\frac{t^{n}}{n !} A^{n} f\right)\right\| .
\end{aligned}
$$

Using that $e^{A t} f$ is $n$-times differentiable in $t$, that

$$
\int_{0}^{t} \mathrm{~d} s \frac{(t-s)^{n-1}}{(n-1) !} e^{A s} A^{n} f,
$$

is the remainder term in the Taylor expansion of $e^{A t} f$, and that for $g \in D(A)$

$$
\left(e^{A s}-1\right) g=\int_{0}^{s} \mathrm{~d} u e^{A u} A g,
$$

if $g \in D(A)$ we obtain the bound

$$
\begin{aligned}
\left\|\frac{n !}{t^{n}} f^{(n, t)}-f\right\|_{n} & =\sum_{j=0}^{n-1}\left\|\frac{n !}{t^{n}} \int_{0}^{t} \mathrm{~d} s \frac{(t-s)^{n-1}}{(n-1) !} \int_{0}^{s} \mathrm{~d} u e^{A u} A^{j+1} f\right\| \\
& +\left\|\frac{n !}{t^{n}}\left(e^{A t} f-f-A f-\cdots-\frac{t^{n}}{n !} A^{n} f\right)\right\| \\
& \leq \mathcal{O}(t)+o(1) .
\end{aligned}
$$


This shows that $f \in \overline{\mathcal{D}}^{n}$. This proves that $\overline{\mathcal{D}}^{n}=D\left(A^{n}\right)$ as required.

Acknowledgments. We thank P. Collet for helpful remarks concerning Theorem 3.8. We have also profited from useful discussions with G. Ben Arous, G. Gallavotti, B. Helffer, V. Jakšić, H.M. Maire, Ch. Mazza, H. Spohn, and A.-S. Sznitman. This work was partially supported by the Fonds National Suisse.

\section{References.}

[Br] Bruck, R.E.: Asymptotic Convergence of Nonlinear Contraction Semigroups on Hilbert Space. J. Funct. Anal. 18, 15 (1975).

[CL] Casher, A. and J.L. Lebowitz: Heat Flow in Regular and Disordered Harmonic Chains. J. Math. Phys. 12, 1701 (1971).

[Da] Davies, E.B.: One-Parameter Semigroups, London, Academic Press (1980).

[FK] Ford, G.W. and M. Kac: On the Quantum Langevin Equation. J. Stat. Phys. 46, 803 (1987).

[FKM] Ford, G.W., M. Kac and P. Mazur: Statistical Mechanics of Assemblies of Coupled Oscillators. J. Math. Phys. 6, 504 (1965).

[GC] Gallavotti, G. and E.G.D. Cohen: Dynamical Ensembles in Stationary States. J. Stat. Phys. 80, 931 (1995).

[GS] Gihman, I.I. and A.V. Skorohod: Stochastic Differential Equations, Berlin, Springer (1972).

[He] Helffer B.: Semi-Classical Analysis for the Schrödinger Operator and Applications, Lecture Notes in Mathematics 1336, Berlin, Springer (1988).

[HM] Helffer B. and A. Mohammed: Caractérisation du Spectre Essentiel de l'Opérateur de Schrödinger avec un Champ Magnétique. Ann. Inst. Fourier 38, 95 (1988).

[HN] Helffer B. and J. Nourrigat: Hypoellipticité Maximale pour des Opérateurs Polynômes de Champs de Vecteurs, Boston, Birkhäuser (1985).

[Hö] Hörmander L.: The Analysis of Linear Partial Differential Operators I-IV, Berlin, Springer (1985).

[Ho] Hopf E.: Ergodentheorie, Berlin, Springer (1970 reprint).

[IW] Ikeda N. and S.Watanabe: Stochastic Differential Equations and Diffusion Processes, Amsterdam, North-Holland (1981).

[JP1] Jakšić, V. and C.-A. Pillet: Ergodic Properties of the non-Markovian Langevin Equation. Lett. Math. Phys. 41, 49 (1997).

[JP2] Jakšić, V. and C.-A. Pillet: Ergodic Properties of Classical Dissipative Systems I. (To appear). Acta Mathematica

[JP3] Jakšić, V. and C.-A. Pillet: Ergodic Properties of Classical Dissipative Systems II (In preparation).

[JP4] Jakšić, V. and C.-A. Pillet: Spectral Theory of Thermal Relaxation. J. Math. Phys. 38, 1757 (1997).

[Ka] Kato, T.: Perturbation Theory for Linear Operators, Berlin, Springer (1980).

[Ne] Nelson, E.: Dynamical theories of Brownian Motion, Princeton, Princeton university press (1980).

[OL] O'Connor, A.J. and Lebowitz, J.L.: Heat Conduction and Sound Transmission in Isotopically Disordered Harmonic Crystals. J. Math. Phys. 15, 692 (1974).

[R-B] Rey-Bellet L.: Steady States and Transport Properties for Mechanical Systems Coupled to Stochastic Heat Baths (In preparation).

[RLL] Rieder, Z., Lebowitz, J.L. and E.Lieb: Properties of a Harmonic Crystal in a Stationary Nonequilibrium State. J. Math. Phys. 8, 1073 (1967).

[RS] Reed M. and B. Simon: Methods of Modern Mathematical Physics I-IV, Boston, Academic Press (1978).

[SL] Spohn, H. and J.L. Lebowitz: Stationary Non-Equilibrium States of Infinite Harmonic Systems. Commun. Math. Phys. 54, 97 (1977).

[Tr] Tropper, M.M.: Ergodic and Quasi-deterministic Properties of Finite Dimensional Stochastic Systems. J. Stat. Phys. 17, 491 (1977).

[Yo] Yosida, K.: Functional Analysis, Berlin, Springer (1980). 